\title{
Respiratory syncytial virus infection exacerbates pneumococcal pneumonia via Gas6/Axl-mediated macrophage polarization
}

\author{
Takehiko Shibata, ${ }^{1}$ Airi Makino, ${ }^{1,2}$ Ruiko Ogata, ${ }^{3}$ Shigeki Nakamura, ${ }^{4,5}$ Toshihiro Ito, ${ }^{3}$ Kisaburo Nagata, ${ }^{2}$ Yoshihiko Terauchi, ${ }^{6,7}$ \\ Taku Oishi, ${ }^{7}$ Mikiya Fujieda, ${ }^{7}$ Yoshimasa Takahashi, ${ }^{1}$ and Manabu Ato ${ }^{1,8}$ \\ 'Department of Immunology, National Institute of Infectious Diseases, Tokyo, Japan. ²Department of Biomolecular Science, Faculty of Science, Toho University, Chiba, Japan. ${ }^{3}$ Department of Immunology, \\ Nara Medical University, Nara, Japan. ${ }^{4}$ Department of Chemotherapy and Mycoses, National Institute of Infectious Diseases, Tokyo, Japan. ${ }^{5}$ Department of Microbiology, Tokyo Medical University, \\ Tokyo, Japan. ${ }^{6}$ Department of Pathology, National Institute of Infectious Diseases, Tokyo, Japan. ${ }^{7}$ Department of Pediatrics, National Hospital Organization Kochi Hospital, Kochi, Japan. ${ }^{8}$ Department of \\ Mycobacteriology, National Institute of Infectious Diseases, Tokyo, Japan.
}

\begin{abstract}
Patients with respiratory syncytial virus (RSV) infection exhibit enhanced susceptibility to subsequent pneumococcal infections. However, the underlying mechanisms involved in this increased susceptibility remain unclear. Here, we identified potentially novel cellular and molecular cascades triggered by RSV infection to exacerbate secondary pneumococcal pneumonia. RSV infection stimulated the local production of growth arrest-specific 6 (Gas6). The Gas6 receptor AxI was crucial for attenuating pneumococcal immunity in that the Gas6/Axl blockade fully restored antibacterial immunity. Mechanistically, Gas6/Axl interaction regulated the conversion of alveolar macrophages from an antibacterial phenotype to an M2-like phenotype that did not exhibit antibacterial activity, and the attenuation of caspase-1 activation and IL-18 production in response to pneumococcal infection. The attenuated IL-18 production failed to drive both NK cell-mediated IFN- $\gamma$ production and local NO and TNF- $\alpha$ production, which impair the control of bacterial infection. Hence, the RSV-mediated Gas6/Axl activity attenuates the macrophage-mediated protection against pneumococcal infection. The Gas6/Axl axis could be a potentially novel therapeutic target for RSV-associated secondary bacterial infection.
\end{abstract}

\section{Introduction}

Globally, respiratory syncytial virus (RSV) is the major cause of respiratory tract infection among humans. Particularly, infants under the age of 2 years are susceptible to RSV infection and the infection often recurs later in life (1). Infants during the first 6 months of life, elderly individuals, and adult patients with respiratory diseases are susceptible to lower respiratory tract infections with RSV $(2,3)$. The RSV infections of the lower respiratory tract account for 64 million cases of pneumonia and approximately 200,000 deaths annually $(4,5)$. Various bacteria, such as Streptococcus pneumoniae, Staphylococcus aureus, non-typable Haemophilus influenzae, and Moraxella catarrhalis frequently colonize in patients with RSV-associated pneumonia (6-8). Furthermore, RSV infection is associated with a higher risk for bacterial pneumonia (9-11). These clinical observations indicate that human RSV infection increases the susceptibility of patients to subsequent bacterial infection.

One possible mechanism underlying enhanced susceptibility to bacterial infection after RSV infection is the attenuated barrier function of the respiratory epithelium. RSV infection is reported to be associated with an increased number of exfoliated epithelial

Conflict of interest: The authors have declared that no conflict of interest exists. Copyright: () 2020, American Society for Clinical Investigation.

Submitted: October 11, 2018; Accepted: February 26, 2020; Published: May 4, 2020.

Reference information: J Clin Invest. 2020;130(6):3021-3037.

https://doi.org/10.1172/JCl125505. cells (12). Additionally, RSV is reported to increase the virulence of pneumococcus bacteria (11). Furthermore, functional changes in neutrophils may also contribute to secondary bacterial infection (10). However, there are limited studies on secondary bacterial infections associated with RSV when compared with those associated with influenza virus (13-15). Other possible mechanisms involved in the enhanced susceptibility to secondary bacterial infections include the attenuation of antibacterial protective immunity. In a previous study, we demonstrated that RSV stimulates the growth arrest-specific 6 (Gas6)/Axl axis, which regulates the antiRSV and antifungal Th1 immune responses $(16,17)$. Mechanistically, Axl, a high-affinity Gas6 receptor, was reported to attenuate the ability of dendritic cells (DCs) to respond to pathogen-associated molecular patterns through the DC-intrinsic Toll-like receptor (TLR) $(18,19)$. This inhibitory effect of Gas6/Axl contributes to the induction of SOCS1 and Twist $(20,21)$. In the mouse model, the Gas6/Axl axis is the key regulator of antibacterial immunity following primary RSV infection. However, the mechanisms underlying the increased susceptibility to subsequent bacterial infection after initial RSV infection have not been elucidated.

In this study, we investigated the role of the RSV-triggered Gas6/Axl axis in secondary S. pneumoniae infection. The serial analysis using gene-deficient mice and blocking antibodies revealed that the Gas6/Axl axis is a crucial regulator of protective immunity against pneumococcal infection. Intriguingly, the mechanisms underlying the Gas6/Axl-mediated immune modu- 


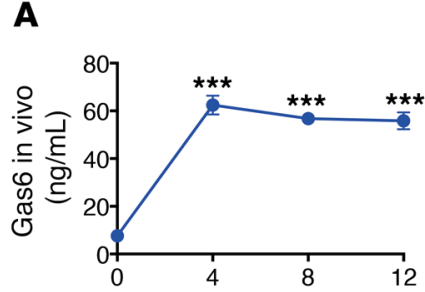

Day after RSV infection

B After RSV DO D4

D4 D8

D12

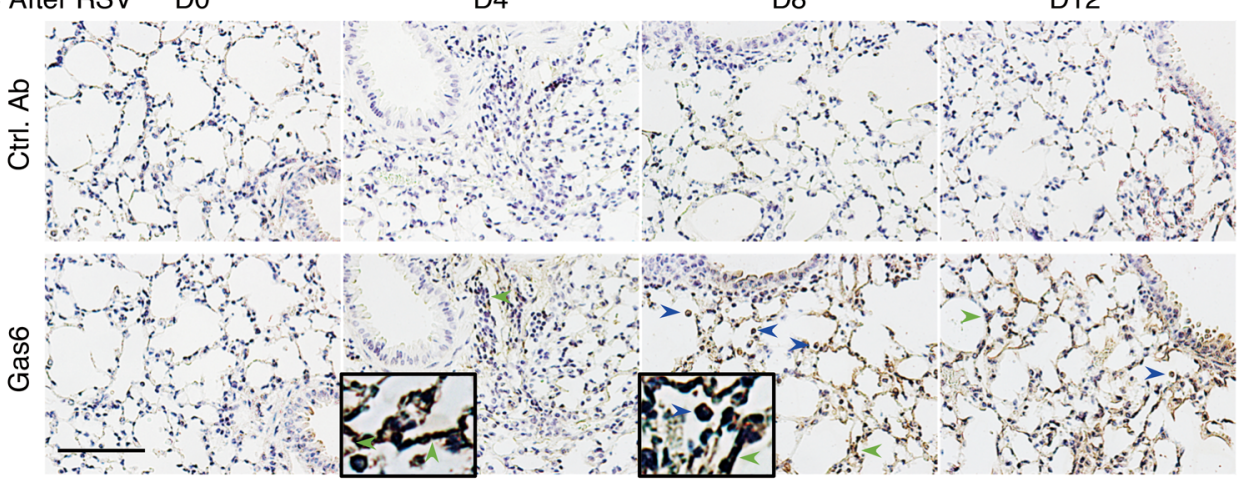

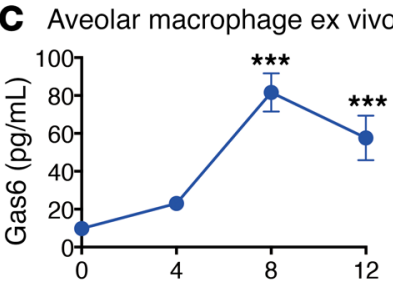

Day after RSV infection
D

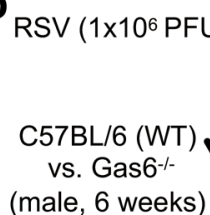

(male, 6 weeks)

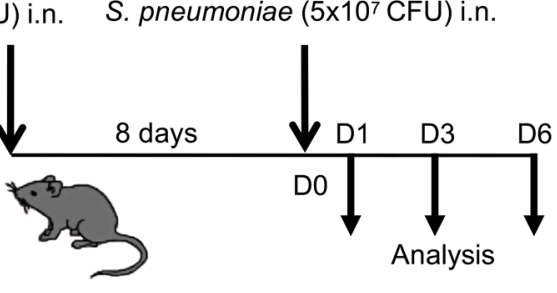

$\mathbf{G}$
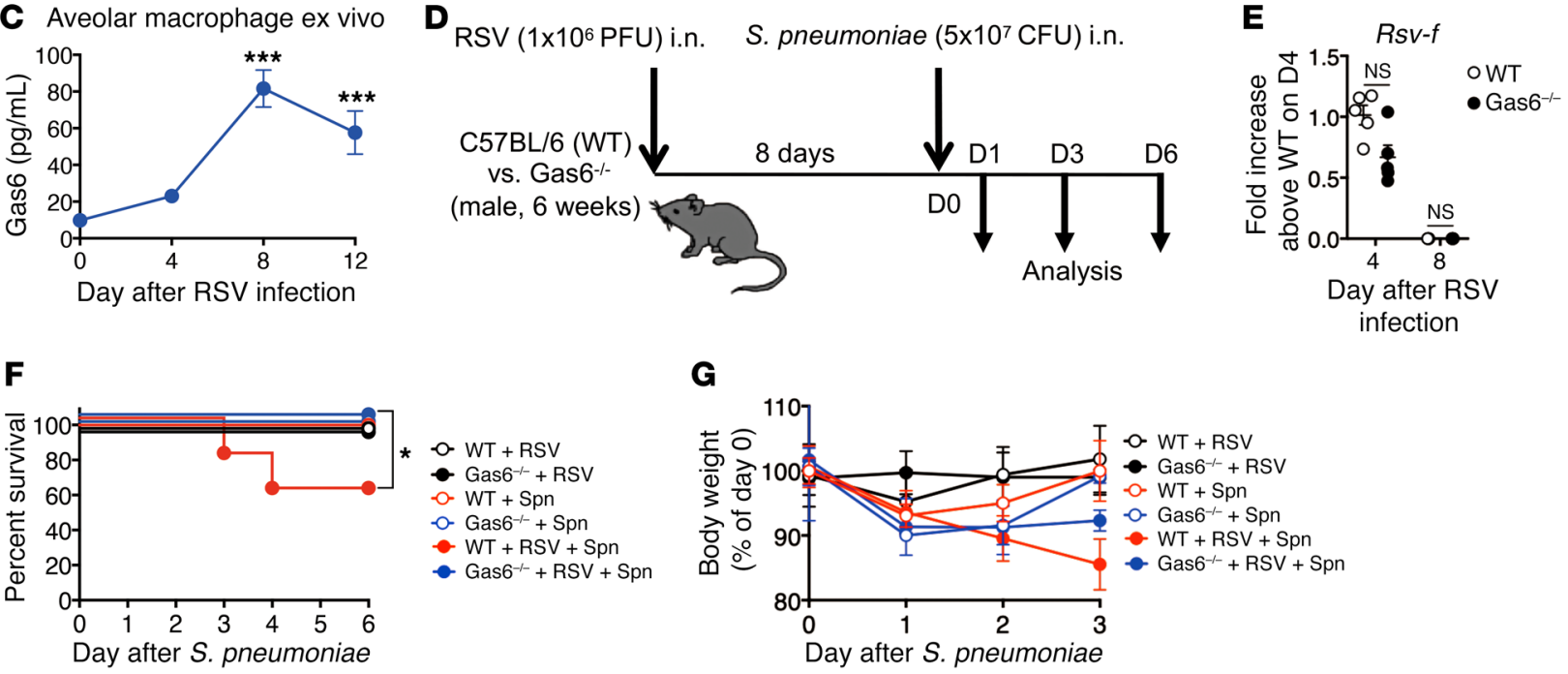

| Spn RSV+Spn

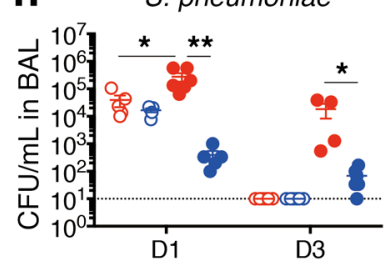

Day after S. pneumoniae

$$
\begin{aligned}
& \text { O WT + Spn } \\
& \text { O Gas6 } 6^{-1-}+\text { Spn } \\
& \text { - } W T+R S V+S p n \\
& \text { - } \mathrm{Gas6}^{-1-}+\mathrm{RSV}+\mathrm{Spn}
\end{aligned}
$$
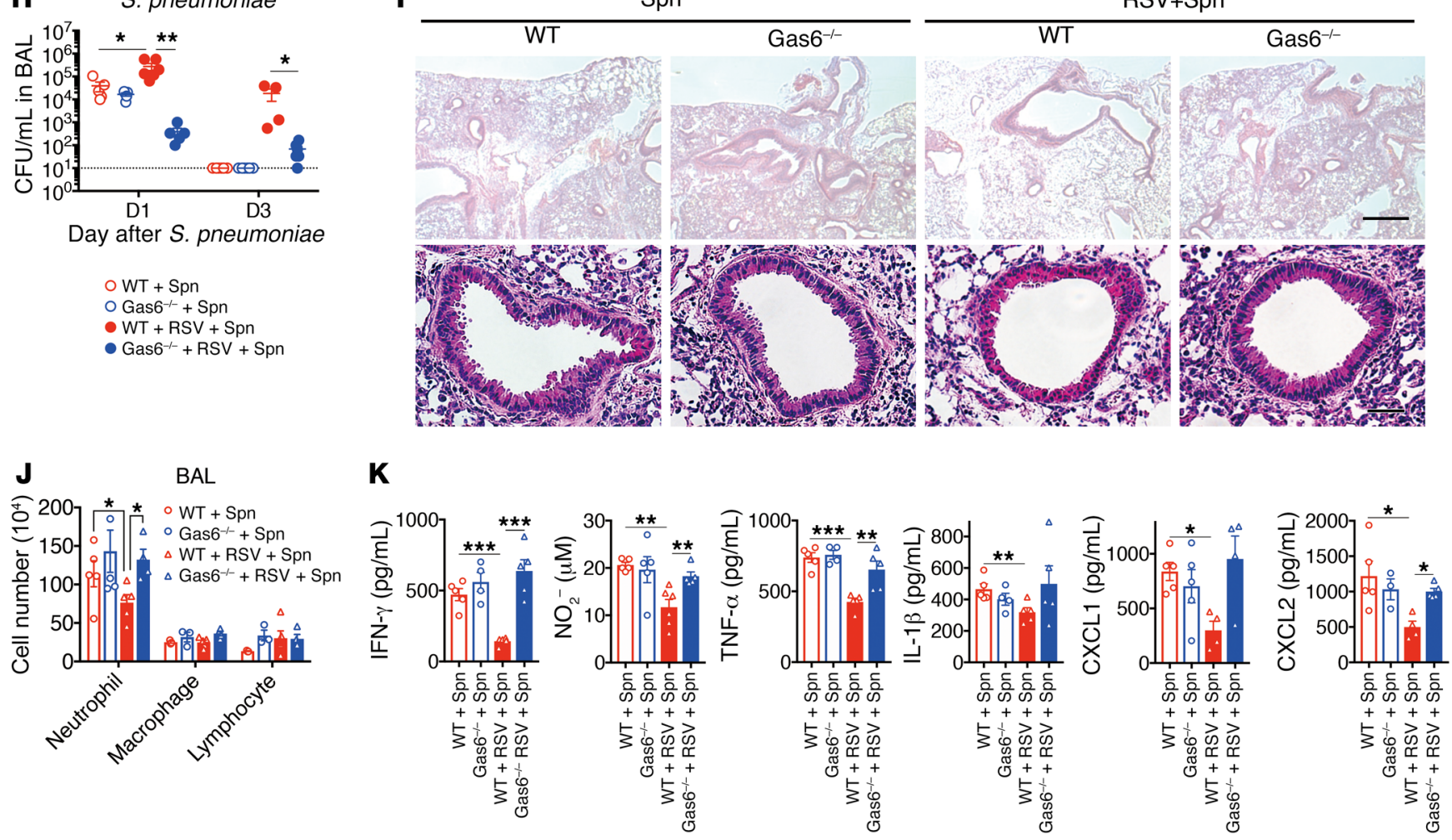
Figure 1. RSV-induced Gas6 enhances susceptibility to S. pneumoniae.

(A) Gas6 levels in the BAL fluid from an RSV-infected lung of a WT mouse. (B) Immunohistochemical analysis (DAB) of Gas6 in the tissue sections from a naive (DO) and RSV-infected lung. Blue arrows indicate Gas6 ${ }^{+}$ macrophages and green arrows indicate $\mathrm{Cas}^{+}$epithelial cells. Scale bar: $200 \mu \mathrm{m}$. (C) Gas6 levels in the culture supernatant of alveolar macrophages isolated from naive and RSV-infected mice on days 4, 8, and 12 after infection. (D) WT and $\mathrm{Gas6}^{-/-}$mice were infected with S. pneumoniae at day 8 after RSV infection and euthanized on days 1, 2, 3, and 6 after 5 pneumoniae infection. (E) Titers of RSV in the whole lung from RSV-infected mice on days 4 and 8 after RSV infection. (F) Changes in the survival rate and (C) body weight of RSV-infected WT or $\mathrm{Cas}^{-/-}$mice after S. pneumoniae infection. (H) Titers of S. pneumoniae in the BAL fluid from RSV-infected mice on days 1 and 3 after 5 . pneumoniae infection. (I) Representative H\&E-stained lung tissue sections on day 1 after S. pneumoniae infection. Scale bars: $200 \mu \mathrm{m}$ (upper), $50 \mu \mathrm{m}$ (lower). (J) Number of neutrophils, macrophages, and lymphocytes in the BAL fluid on day 1 after 5 . pneumoniae infection. (K) The levels of IFN- $\gamma$, NO, TNF- $\alpha$, IL-1 $1 \beta, C X C L 1$, and CXCL2 in the BAL fluid from WT and Cas6 ${ }^{-1-}$ mice on day 1 after S. pneumoniae infection. The data are expressed as mean \pm SEM; $n=10$ (F and $\mathbf{G}$ ), $n=4-6$ (except for $\mathbf{F}$ and $\mathbf{G}$ ). Representative results from 2 independent experiments are shown. The following statistical tests were used: 1-way ANOVA (A, C, H, J, and K), 2-tailed Student's $t$ test (E), and Gehan-Breslow-Wilcoxon test $(\mathbf{F}) .{ }^{*} P<0.05 ;{ }^{* *} P<0.01 ;{ }^{* *} P<0.001$.

lation were different from the mechanisms previously reported to be involved in the anti-RSV and antifungal immune responses. Gas6/Axl converted the resident alveolar macrophage from an antibacterial phenotype to an M2-like phenotype that did not exhibit antibacterial activity. This phenotypic change inhibits the downstream effector cascades from clearing the bacterial burden. These findings provide potentially novel mechanistic insights for developing therapeutic strategies for RSV-associated secondary bacterial infections.

\section{Results}

Gas6 increases the susceptibility to $S$. pneumoniae infection. We infected the C57BL/6 mice with the RSV A2 strain by i.n. injection. The replication of RSV was observed in the bronchoalveolar lavage (BAL) fluid. However, RSV infection did not increase the number of exfoliated epithelial cells on day 8 after RSV infection (Supplemental Figure 1, A and B; supplemental material available online with this article; https://doi.org/10.1172/JCI125505DS1). RSV infection induced Gas6 protein expression in the BAL fluid by day 4 and maintained the expression levels until day 12 (Figure 1A). Immunohistochemical analysis of the lung sections revealed the expression of Gas6 protein in the alveolar epithelial cells by day 4. Additionally, the expression of Gas6 was detected in the alveolar macrophages at days 8 to 12 (Figure 1B). The expression of Gas 6 in the isolated alveolar macrophages, especially those isolated after day 8 , was confirmed in vitro (Figure 1C). To evaluate the effect of Gas6 on the RSV-mediated attenuation of bacterial clearance, we first developed a mouse model. In this model, the mice preexposed to RSV exhibited attenuated clearance of subsequent pneumococcal infection. The mice were injected (i.n.) with S. pneumoniae at day 8 after RSV infection. The mice were euthanized at days 1 and 6 after $S$. pneumoniae infection (Supplemental Figure 2A). In this model, the initial RSV infection exacerbated the morbidity and mortality of subsequent infection by $S$. pneumoniae (Supplemental Figure 2, B and C), which mimicked the clinical observations (22-24). The primary S. pneumoniae infection was cleared by day 3 after infection, but the clearance was delayed in mice preexposed to RSV (Supplemental Figure 2D). S. pneumoniae infection transiently induced peribronchial accumulation of various inflammatory cells, such as neutrophils, interstitial macrophages, $\mathrm{CD} 4^{+} \mathrm{T}$ cells, $\mathrm{CD} 8^{+} \mathrm{T}$ cells, and NK cells at day 1 after $S$. pneumoniae infection. The mice preexposed to RSV did not exhibit peribronchial accumulation of inflammatory cells (Supplemental Figure 2, E and F). Similarly, preexposure to RSV significantly suppressed IFN- $\gamma$ production following pneumococcal infection (Supplemental Figure 2G). Further, preexposure to RSV attenuated the IFN- $\gamma$-induced secretion of effector cytokines, NO, and CXCL1 and CXCL2, but not leukotriene B4, which are powerful neutrophil chemoattractants, after infection. These results suggest that prior RSV infection may increase the susceptibility to pneumococcal infection by suppressing the immune responses in the developed mouse model.

RSV infection promotes the expression of Gas6 and its receptor Axl, which majorly contributes to the RSV-induced immune responses under several conditions $(17,25)$. Thus, we first assessed the role of the Gas6/Axl axis in increasing the susceptibility to secondary bacterial infection after preexposure to RSV (Figure 1D). The virus titers on days 4 and 8 after RSV infection were not significantly different between Gas6-deficient $\left(\right.$ Gas $\left.^{-/}\right)$and WT mice (Figure 1E). The RSV-mediated suppression of antibacterial immunity, which was determined based on the survival rate, body weight loss, and bacterial burden, was mitigated in the $\mathrm{Gas}^{-/-}$mice compared with mice preexposed to RSV upon pneumococcal infection (Figure 1, F-H). The restored antibacterial immunity in the $\mathrm{Gas}^{-/}$mice was correlated with the accumulation of inflammatory cells in the peribronchial region and BAL fluid (Figure 1, I and J). The increased numbers of inflammatory cells in the Gas $6^{-/}$mice promoted the production of IFN- $\gamma$, NO, TNF- $\alpha$, CXCL1, and CXCL2 (Figure 1K).

To further confirm the suppressive role of RSV-induced Gas6 in this mouse model, we administered (i.n.) the recombinant Gas6 (rGas6) to naive WT mice for 4 days before S. pneumoniae infection (Figure 2A). This time frame was selected to mimic the presence of endogenous Gas 6 for 4 days before pneumococcal infection (Figure 1, A and B). Similar to the mice preexposed to RSV, rGas6-treated mice exhibited significantly high susceptibility to S. pneumoniae infection (Figure 2, B and C). The rGas6-treated and RSV-infected groups exhibited high levels of bacterial burdens (Figure 2D). Contrastingly, fewer inflammatory cells were recruited into the peribronchial region and BAL fluid after rGas6 treatment (Figure 2, E and F). Prior Gas6 administration markedly reduced the number of neutrophils, whereas the number of macrophages and lymphocytes remained largely intact. The reduction in cell numbers was also correlated with low detection of several immune mediators in the rGas6-treated mice (Figure 2G). These results indicate that Gas6, which is produced upon RSV infection, plays a critical role in attenuating antibacterial immunity.

Axl increases the susceptibility to S. pneumoniae infection. Among the 3 TAM receptors (Tyro3, Axl, and Mert), Gas6 binds Axl with the highest affinity (26-28). Next, we investigated the role of Axl as an immunoregulator in our mouse model. Axl was constitutively expressed in the lung, especially in the alveolar macrophages. The mRNA and protein expression levels of Axl increased upon 
A<smiles>CC[GeH2]C1CCC1</smiles>

RSV $\left(1 \times 10^{6} \mathrm{PFU}\right)$ i.n.

rGas6 (1 $\mu \mathrm{g} /$ i.n. $)$

S. pneumoniae $\left(5 \times 10^{7} \mathrm{CFU} /\right.$ i.n. $)$

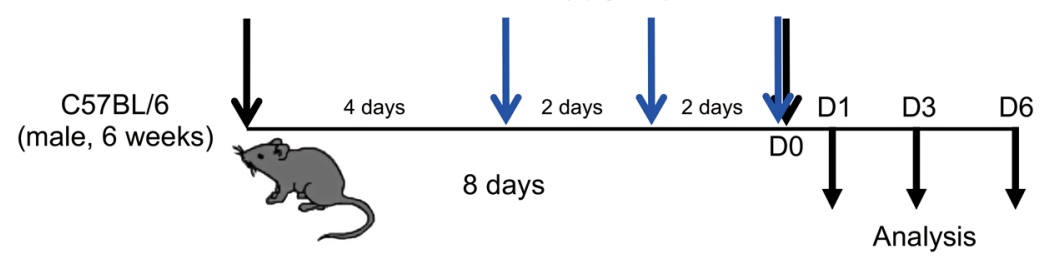

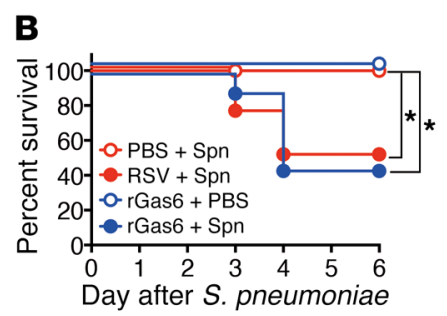

C

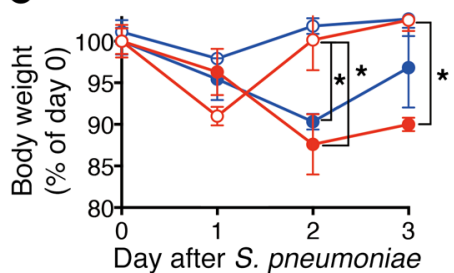

- - PBS + Spn - RSV + Spn - rGas6 + PBS - rGas6 + Spn

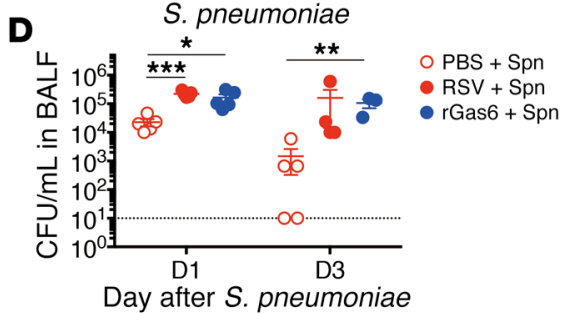

Day after $S$. pneumoniae
E $\mathrm{PBS}+\mathrm{Spn}$

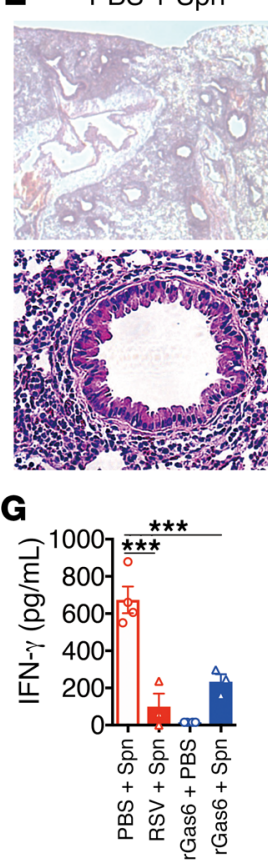

Spn + RSV

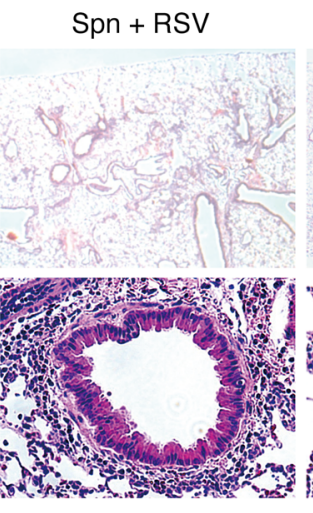

$$
\text { PBS + rGas6 }
$$

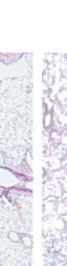

$\mathrm{Spn}+\mathrm{rGas} 6$

$\mathbf{F}$

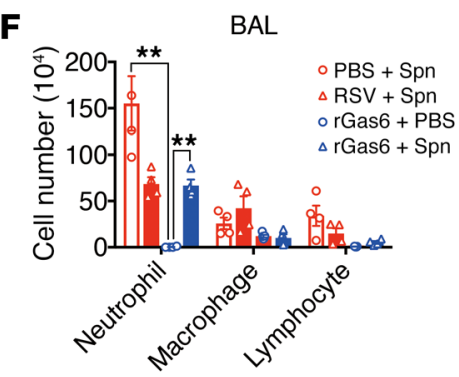

Figure 2. Exogenous Gas6 increases susceptibility to S. pneumoniae infection following RSV infection. (A) Mice were infected with RSV or treated with recombinant Gas6 (rGas6; $1 \mu \mathrm{g} /$ dose, 3 doses) before S. pneumoniae infection on day 8 after RSV infection. Animals were euthanized for analysis on days 1,2 , and 3 after $S$. pneumoniae infection. (B) Changes in the survival rate and (C) body weight after S. pneumoniae infection. (D) Titers of S. pneumoniae in the BAL fluid from RSV-infected or rGas6-injected mice on days 1 and 3 after S. pneumoniae infection. (E) Representative H\&E-stained lung tissue sections on day 1 after $S$. pneumoniae infection. Scale bars: $200 \mu \mathrm{m}$ (upper), $50 \mu \mathrm{m}$ (lower). (F) Numbers of inflammatory cells in the BAL fluid from both treatment groups on day 1 after S. pneumoniae infection. (C) The levels of IFN- $\gamma$, NO, TNF- $\alpha$, IL-1 $\beta$, CXCL1, and CXCL2 in the BAL fluid from both treatment groups on day 1 after $S$. pneumoniae infection. The data are expressed as the mean $\pm S E M ; n=10$ (B and $\mathbf{C}$ ), $n=4-6$ (except for $\mathbf{B}$ and $\mathbf{C}$ ). Representative results from 2 independent experiments are shown. The following statistical tests were used: Gehan-Breslow-Wilcoxon test (B), 1-way ANOVA (C, D, F, and G). ${ }^{*} P<0.05 ;{ }^{* *} P<0.01 ;{ }^{* *} P<0.001$.

RSV infection (Figure 3, A-C), which concurred with the results of our previous study (16). Additionally, we observed that there were 2 populations of alveolar macrophages that exhibited differential expression of Siglec-F (Figure 3D). The alveolar macrophages that exhibited high Siglec-F expression were tissue-resident alveolar macrophages (TR-AMs), whereas those exhibiting low Siglec-F expression were monocyte-derived macrophages (Mo-AMs) (29). Interestingly, the TR-AMs exhibited upregulated Axl expression, whereas the Mo-AMs exhibited downregulated Axl expression (Figure 3D). The flow cytometric analysis revealed that the number of Axl-expressing TR-AMs was significantly higher than the number of Axl-expressing Mo-AMs, interstitial macrophages, DCs, and neutrophils in the lung, especially on day 8 after RSV infection (Figure 3E and Supplemental Figure 3A).

To evaluate the role of Axl in bacterial immunity after RSV infection, the responses of $\mathrm{Axt}^{-/}$mice were compared with WT 
mice in our model. The RSV-mediated suppression of antibacterial immunity in the $A x l^{-/-}$mice, which was evaluated based on the survival rate, body weight loss, and bacterial burden, was mitigated compared with WT mice preexposed to RSV (Figure 3, F-H). The restored protection was correlated with the accumulation of inflammatory cells in the peribronchial region and BAL fluid of $A x \mathrm{I}^{-/-}$mice (Figure 3, I and J). The increased numbers of inflammatory cells in the $A x l^{-/-}$mice promoted the production of IFN- $\gamma, \mathrm{NO}$, TNF- $\alpha$, CXCL1, and CXCL2 (Figure 3K). These results suggest that the Gas6/Axl axis in the alveolar macrophages, especially resident alveolar macrophages, is involved in severe pneumococcal infection after RSV infection. Furthermore, these results were consistent with those obtained using the $\mathrm{Gas}^{-/-}$mice.

Axl inhibition mitigates the RSV-mediated immune suppression against pneumococcal infection. The above findings also suggest that the Gas6/Axl axis may be a drug target for preventing exaggerated bacterial infection. This hypothesis was tested using the anti-Axl blocking $\mathrm{mAb}$ and an Axl-specific small molecule inhibitor, BGB324 (also known as R428). We initially assessed the effect of i.p. administration of anti-Axl blocking mAb into the RSV-infected WT mice. The mice were treated with the anti-Axl $\mathrm{mAb}$ from days 0 to 6 at 2-day intervals before the $S$. pneumoniae infection at day 0 (Figure 4A). Despite the prior RSV infection, the anti-Axl mAb-treated mice showed a protective ability against pneumococcal infection compared with mice without prior RSV infection (Figure 4, B and C). The improved antibacterial immunity in the anti-Axl mAb-treated mice was also reflected in the reduction of bacterial burdens (Figure 4D) and the improvement of airway inflammation, including neutrophil infiltration (Figure 4, E and F). Finally, anti-Axl mAb treatment restored the levels of IFN- $\gamma$ to levels observed in control IgG-treated mice preexposed to RSV (Figure 4G). These results suggest that the Gas6/Axl axis is crucial for the RSV-induced suppression of anti-pneumococcal immunity.

BGB324 specifically inhibits the catalytic and procancerous activities of Axl, but does not affect the binding between Gas6 and Axl (30). The results of Axl activity inhibition by BGB324 treatment concurred with the findings of anti-Axl mAb treatment (Supplemental Figure 4, A-G). The inhibition of Axl restored the protective immunity to pneumococcal infection. Thus, the Gas6/ Axl axis is a promising drug target for reducing the bacterial burden upon secondary bacterial infection.

Axl on the alveolar macrophages but not on the epithelial cells promotes RSV-mediated immune suppression against pneumococcal infection. Axl is expressed not only on WT resident alveolar macrophages, but also on the epithelial cells in the lung (Figure 3B and Supplemental Figure 3, A-C). To identify the cells that are responsible for severe secondary pneumococcal infection, bone marrow chimeric mice were constructed (Figure 5A). When the WT mice were transplanted with the $A x^{-/-}$bone marrow cells, the survival rate, body weight loss, bacterial clearance, neutrophil infiltration, and IFN- $\gamma$ production were significantly restored to the control levels even in the group exposed to secondary bacterial infection (Figure 5, B-G). However, this recovery was not observed in the $A x l^{-/-}$mice transplanted with bone marrow from WT mice. Intriguingly, transplanting the bone marrow from $\mathrm{Gas}^{-/-}$mice into the WT mice partially restored the survival rate, body weight loss, bacterial clearance, the number of neutrophils, and IFN- $\gamma$ pro- duction (Supplemental Figure 5, A-G). These results suggest that Gas6 produced by the macrophages and epithelial cells engages Axl on the alveolar macrophages, which triggers the exacerbation of pneumococcal infection.

Alveolar macrophages are required for pneumococcal protection. Protection against pneumococcal infection is mediated by several effector cells, including alveolar macrophages (31). Our results demonstrated that the resident alveolar macrophages are involved in the secondary pneumococcal infection after RSV infection. To confirm the protective role of alveolar macrophages in our pneumococcal infection model, the alveolar macrophages were depleted by clodronate liposome treatment for 2 days before pneumococcal infection (Figure 6A). Administration (i.n.) of clodronate liposome preferentially depleted the alveolar macrophages, but not the interstitial macrophages or DCs (Figure 6B and Supplemental Figure 6). Importantly, the depletion of alveolar macrophages exacerbated the pneumococcal disease, which was evaluated based on reduced survival rate, severe body weight loss, and increased bacterial burden (Figure 6, C-E). The alveolar macrophage-depleted mice exhibited decreased peribronchial accumulation of inflammatory cells and production of inflammatory cytokines in the BAL fluid (Figure 6, F-H). This suggested that the alveolar macrophage is a key initiator of inflammatory responses against the pneumococcal bacterial infection.

$R S V$-triggered Gas6/Axl axis polarizes alveolar macrophage phenotype. Because the resident alveolar macrophages were demonstrated to be "conductor" cells in our model, the phenotype of alveolar macrophages was investigated after RSV infection. The alveolar macrophages from naive mice exhibited antibacterial activity against $S$. pneumoniae in vitro (Figure 7, A and B). However, the macrophages generated on day 8 after RSV infection exhibited low antibacterial activity. The macrophage phenotype exhibited marked polarization during the 8 days after RSV infection (Figure 7, C and D). At the time of RSV infection, the resident alveolar macrophages exhibited a typical MO phenotype lacking the M1 markers (inducible NO synthase gene, Nos2) and M2 markers (genes encoding arginase-1, Arg1, and found in inflammatory zone; Fizz1, and CD206). However, these macrophages acquired the M2 phenotype at day 8 after RSV infection. Based on these results, the resident macrophages and macrophages isolated on day 8 after RSV infection were designated as M0-like and M2-like macrophages, respectively. Importantly, M0-like to M2-like polarization was suppressed in the $\mathrm{Gas}^{-{ }^{--}}$mice, which indicated that this process is dependent on Gas6 expression. Contrastingly, the phenotypic transition of macrophages is also mediated by the Th2 cytokines during RSV infection (32). In the lung, the expression of IL-13, which is one of the inducers of M2-like macrophages, was detected from days 4 to 12 after RSV infection (Figure 7E).

To examine the role of macrophage phenotypic conversion by the RSV-induced Gas6/Axl axis in macrophage-mediated antibacterial immunity, the RSV-infected mice were intratracheally (i.t.) administered alveolar macrophages isolated at day 0 (MO-like) or day 8 (M2-like) after RSV infection (Figure 8, A and B). The antibacterial immunity, which was evaluated based on the survival rates, body weight loss, and bacterial numbers in the BAL fluid of mice receiving the M2-like macrophages was not affected, whereas that of mice receiving the MO-like macrophages was restored 
A
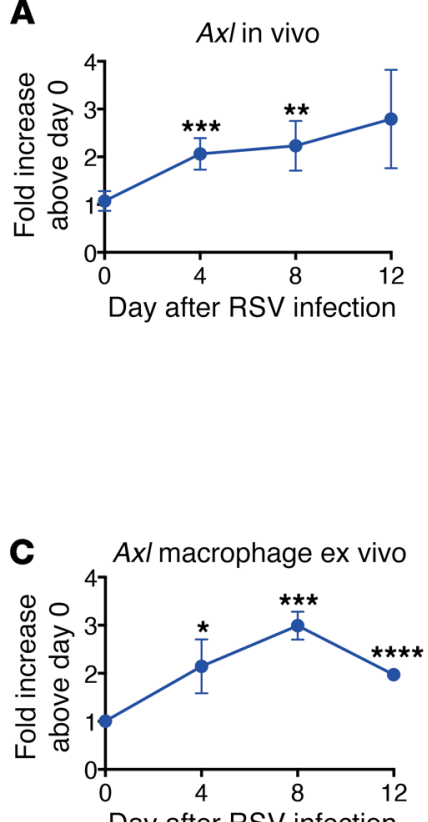

Day after RSV infection

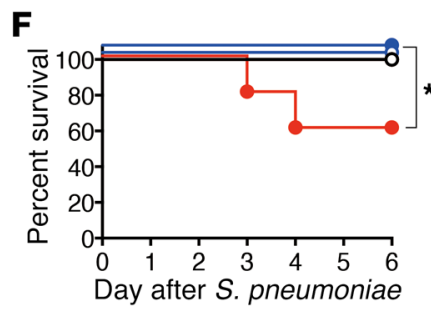

\begin{tabular}{lll} 
I Spn & \\
\hline WT & & $\mathrm{Ax}^{-1-}$
\end{tabular}
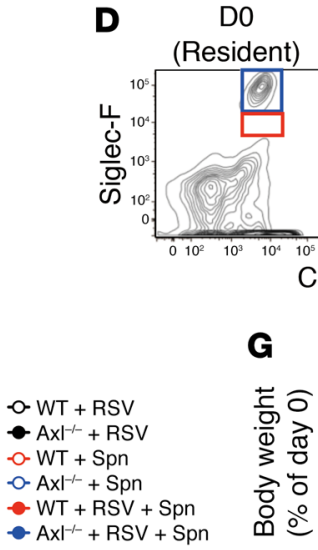

B After RSV D0

D4

D8

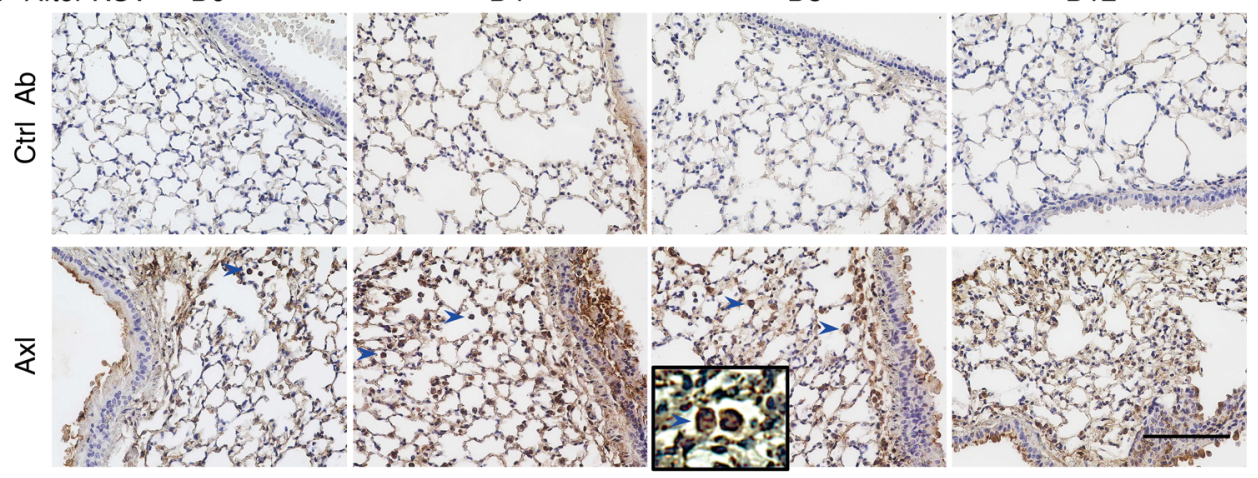

CD11b

D8

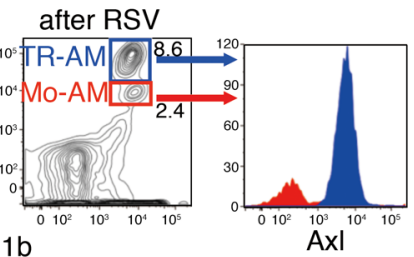

E $\quad \mathrm{Axl}^{+}$cell

G

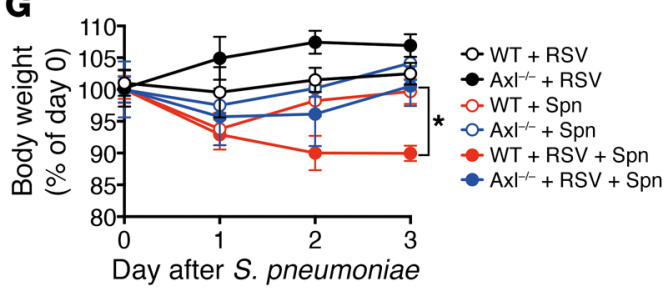

\begin{tabular}{ll} 
RSV+Spn \\
\hline WT
\end{tabular}
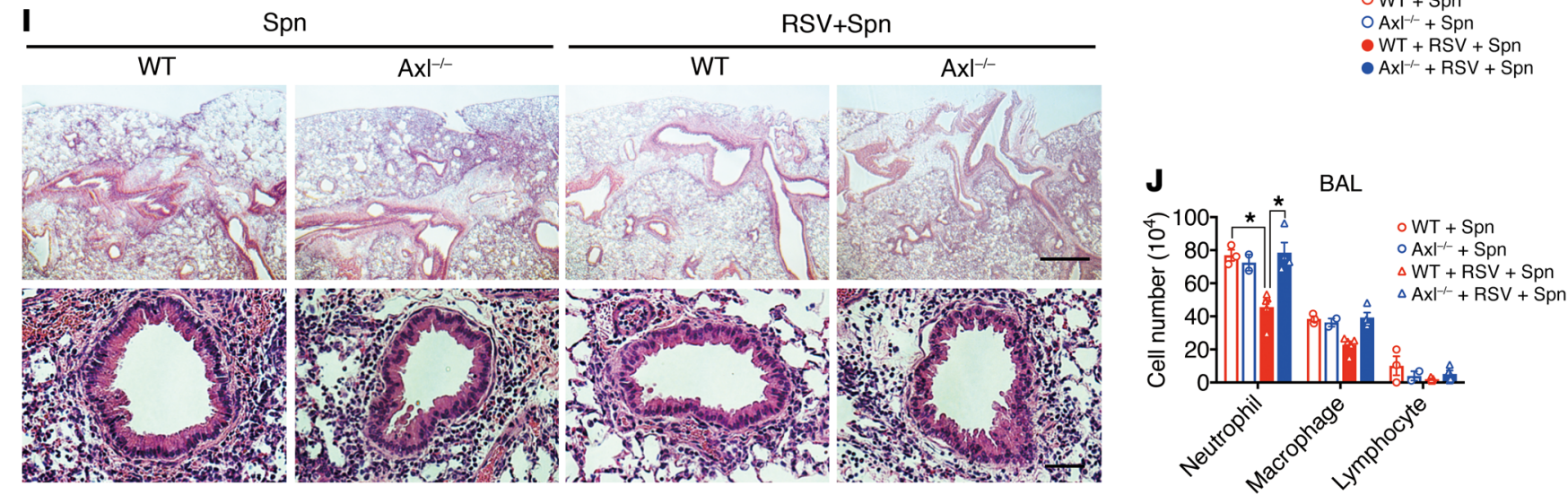

S. pneumoniae

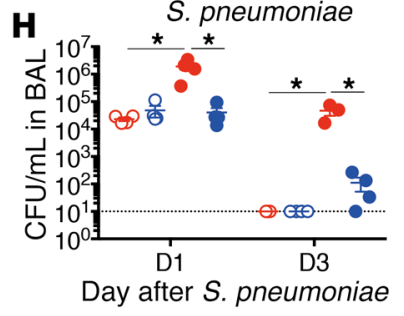

OWT + Spn

$\mathrm{OAxl}{ }^{-1-}+\mathrm{Spn}$

- $A x^{-1-}+\mathrm{RSV}+\mathrm{Spn}$
- $W T+R S V+S p n$
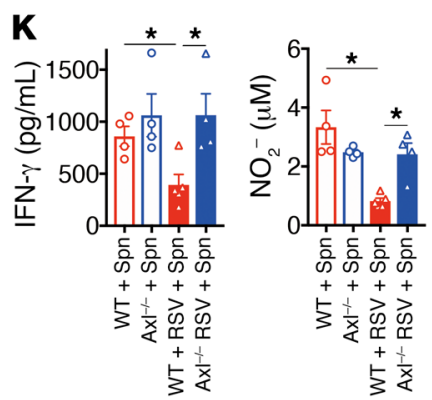
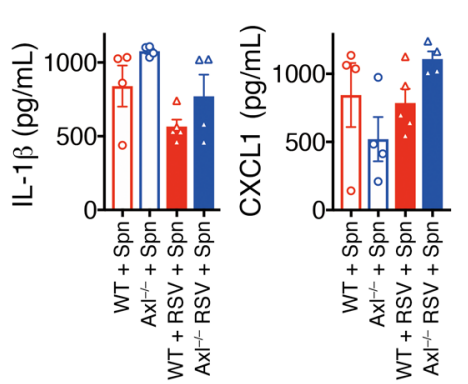
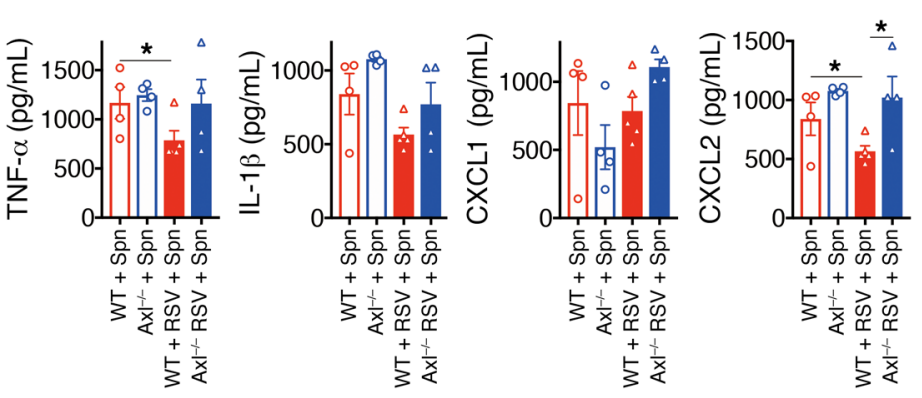
Figure 3. Axl is involved in promoting susceptibility to $S$. pneumoniae after RSV infection. (A) Axl levels in whole lung from an RSV-infected WT mouse. (B) Immunohistochemical analysis (DAB) of Axl in the lung tissue sections from naive (DO) and RSV-infected mice. Blue arrows indicate $\mathrm{Axl}^{+}$macrophages. Scale bar: $200 \mu \mathrm{m}$. (C) Axl expression in the alveolar macrophages isolated from naive and RSV-infected mice on days 4, 8 , and 12 after infection. (D) Axl expression in the tissue-resident alveolar macrophages (TR-AM) and monocyte-derived alveolar macrophages (Mo-AM) from naive and RSV-infected mice at day 8 after RSV infection. (E) Temporal changes in the number of $\mathrm{Axl}^{+}$cells after RSV infection. (F) Changes in the survival rate and (C) body weight of RSV-infected WT or AxI $\mathrm{I}^{-1-}$ mice after S. pneumoniae infection. (H) Titers of S. pneumoniae in the BAL fluid from RSV-infected mice on days 1 and 3 after $S$. pneumoniae infection. (I) Representative $\mathrm{H} \& \mathrm{E}$-stained lung tissue sections on day 1 after S. pneumoniae infection. Scale bars: $200 \mu \mathrm{m}$ (upper), $50 \mu \mathrm{m}$ (lower). (J) The number of neutrophils, macrophages, and lymphocytes in the BAL fluid on day 1 after 5 . pneumoniae infection. (K) The levels of IFN- $\gamma$, NO, TNF- $\alpha$, IL-1 $\beta, C X C L 1$, and CXCL2 in the BAL fluid from WT and $A x^{-1-}$ mice on day 1 after $S$. pneumoniae infection. The data are expressed as mean \pm SEM; $n=10$ ( $\mathbf{F}$ and $\mathbf{G}$ ), $n=4-5$ (except for $\mathbf{F}$ and $\mathbf{G}$ ). Representative results from 2 independent experiments are shown. The following statistical tests were used: One-way ANOVA (A, E, G, H, J, and $\mathbf{K}$ ) and Gehan-BreslowWilcoxon test $(\mathbf{F}) .{ }^{*} P<0.05$; ${ }^{* *} P<0.01$; ${ }^{* *} P<0.001$; ${ }^{* * *} P<0.0001$.

(Figure 8, C-F). The administration (i.t.) of M0-like macrophages enhanced neutrophil infiltration into the peribronchial regions and local production of IFN- $\gamma$ (Figure 8G). These results suggested that the RSV-initiated phenotypic conversion of MO-like macrophages during secondary pneumococcal infection is the primary cause of severe bacterial infection.

RSV-polarized macrophages exhibited low IL-18 production upon secondary bacterial infection. An important potential function of the resident alveolar macrophages during bacterial infection is the production of proinflammatory cytokines, such as IL-18 that is not produced after M2 polarization (33). The inhibition of the Gas6/Axl axis by Gas6 and Axl deficiency or the addition of antiAxl blocking $\mathrm{mAb}$ or Axl inhibitor restored IL-18 production upon secondary pneumococcal infection (Figure 9A). Similarly, the supplementation of exogenous rGas6 suppressed IL-18 production. The depletion of nonpolarized resident alveolar macrophages reduced IL-18 production, which indicated that the resident alveolar macrophages have a critical role in IL-18 production upon bacterial infection. Finally, the inhibition of IL-18 by blocking $\mathrm{mAb}$ decreased and supplementation of exogenous rIL-18 enhanced cytokine (IFN- $\gamma$ and TNF- $\alpha$ ) and NO production at the local sites (Figure 9, B and C). Thus, these data indicated that IL-18 is a regulator of downstream cytokines in this mouse model, which was also observed in other models.

To further explore the mechanism underlying the inhibition of IL-18 production in the RSV-polarized macrophages during bacterial infection, we isolated the M2-like macrophages and stimulated them with S. pneumoniae for 3 hours (Figure 9D). The isolated alveolar macrophages could produce IL-18 upon bacterial stimulation, which was inhibited when the cells were polarized by preexposure to RSV. This process was dependent on Gas6. Combined with the macrophage depletion studies, these data reveal that the alveolar macrophages are an important cellular source of IL-18 during pneumococcal infection. Furthermore, the results suggest that the RSV-driven polarization of alveolar macrophages is the key event for attenuating antibacterial immunity. Consistent with the pre- vious data (Figure 9, A and B), the polarization was dependent on Gas6 expression in that Gas6 deficiency restored IL-18 production from alveolar macrophages (Figure 9E). To elucidate the molecular mechanisms underlying the bacterium-induced IL-18 production by macrophage polarization, we examined the caspase- 1 and NF- $\mathrm{KB}$ pathways, which are both required for IL-18 production. As shown in Figure 9F, the alveolar macrophages from the mice preexposed to RSV exhibited decreased $\mathrm{p} 10$ expression and enhanced I $\mathrm{B}$ expression upon bacterial stimulation. This indicated that the activation of both caspase-1 and NF- $\mathrm{KB}$ pathways were compromised with prior RSV conditioning of alveolar macrophages.

$R S V$-mediated immune regulation leads to impaired IFN- $\gamma$ production from NK cells. An important function of IL-18 is to stimulate IFN- $\gamma$, which plays an important role in protection against intracellular bacteria by regulating the production of various effector molecules, such as NO and TNF- $\alpha(34,35)$. To further dissect the downstream cascade of IL-18, we examined IFN- $\gamma$ production after bacterial infection with or without preexposure to RSV. We identified that NK cells were the primary IFN- $\gamma$ producers after bacterial infection. Notably, IFN- $\gamma$ production was markedly inhibited by preexposure to RSV. However, the suppression was mitigated upon treatment with the anti-Axl blocking $\mathrm{mAb}$ (Figure 10, A-C). The important contribution of NK cell-derived IFN- $\gamma$ was further confirmed by treating the mice with NK celldepleting mAbs before bacterial infection. The depletion of NK cells almost completely inhibited the production of IFN- $\gamma$ in the lung after $S$. pneumoniae infection, which resulted in decreased survival rate, body weight, bacterial clearance, and airway inflammation (Supplemental Figure 7, A-G). Along with the NK cells and macrophages, neutrophils also contribute to bacterial clearance $(36,37)$. The depletion of neutrophils using the antiLy6G mAb significantly reduced the survival rate, body weight, bacterial clearance, and airway inflammation. This suggested that neutrophils were also essential in our model (Supplemental Figure 8, A-F). However, treatment with anti-Ly6 G mAb did not affect the production of IFN- $\gamma$ due to the inhibition of neutrophilderived IFN- $\gamma$ production on day 1 after $S$. pneumonia infection (Supplemental Figure 8, G and H). Furthermore, the depletion of neutrophils did not affect the survival rate when compared with the group of mice with secondary bacterial infection after RSV infection. The RSV-associated activation of Gas6/Axl cascades resulted in decreased IFN- $\gamma$ production in the NK cells, which led to secondary pneumococcal infection.

\section{Discussion}

In this study, we investigated the molecular basis of the severity of secondary pneumococcal infection that follows RSV infection. The changes in the epithelium during RSV infection are reported to facilitate secondary bacterial infection (12). However, there was no marked increase in the number of exfoliated epithelial cells on day 8 after RSV infection in the mouse model developed in this study (Supplemental Figure 1, A and B). Alternatively, the Gas6/Axl axis induced by RSV infection converted the macrophages from an antibacterial (resident) to an M2-like phenotype that did not exhibit antibacterial activity, with attenuated production of macrophage-derived IL-18 and NK cell-derived IFN- $\gamma$. This was followed by the reduction of NO, TNF- $\alpha$, and 
A $\operatorname{RSV}\left(1 \times 10^{6} \mathrm{PFU}\right) \quad$ Axl blocking mAb $(5 \mu \mathrm{g} / \mathrm{i} . \mathrm{p}$.$) \quad S. pneumoniae \left(5 \times 10^{7} \mathrm{CFU}\right)$

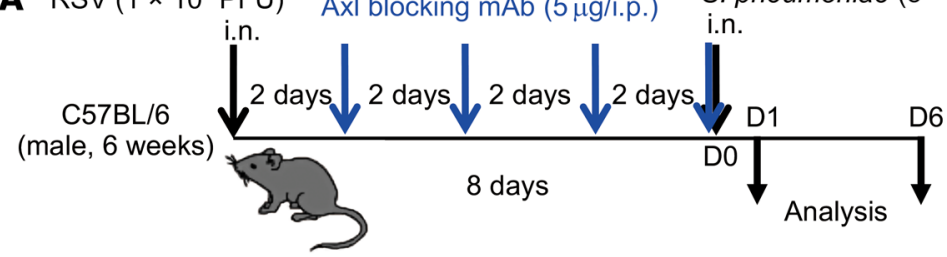

B

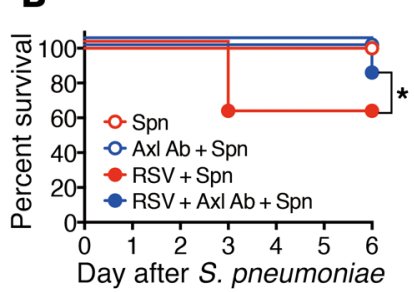

C

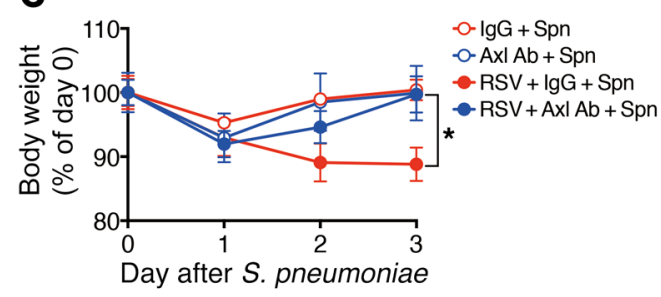

D

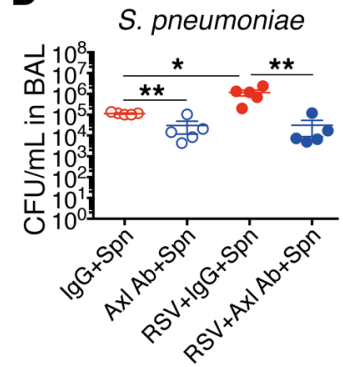

E

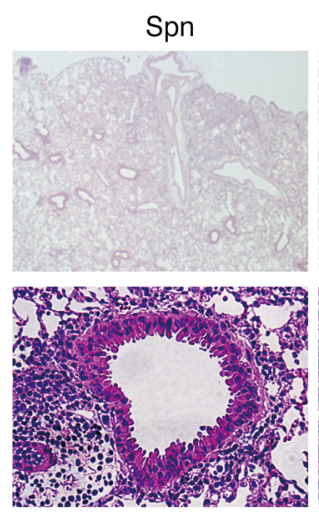

$A x \mid A b+S p n$
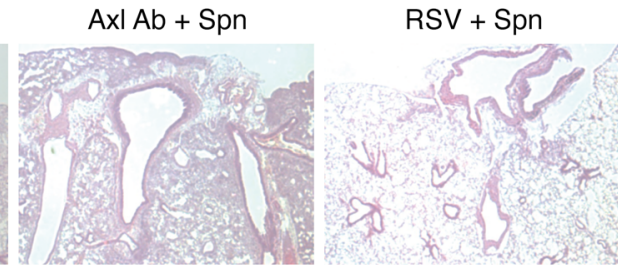

$R S V+A x l A b+S p n$
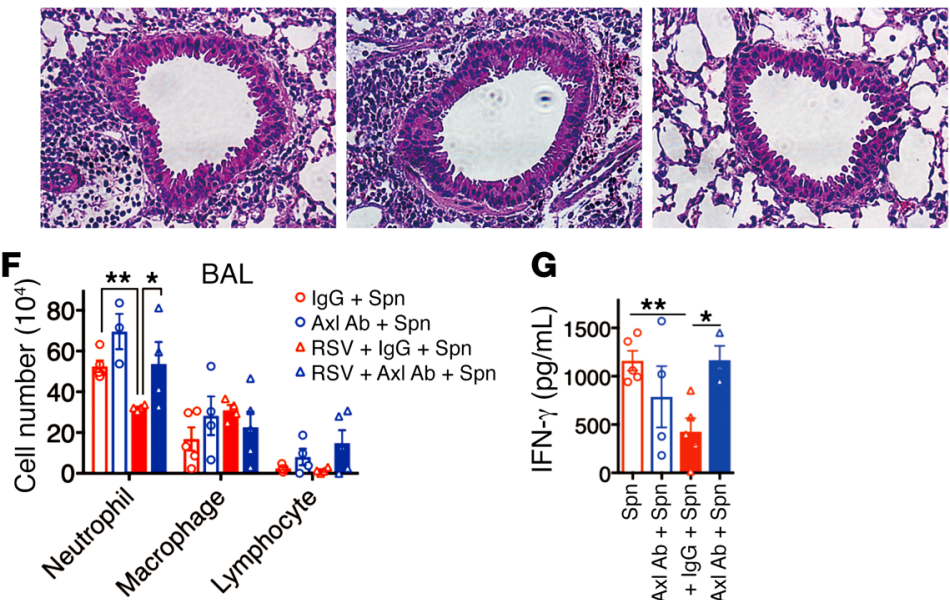

G

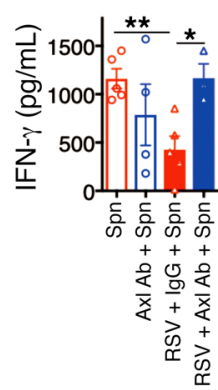

Figure 4. AxI receptor blockade attenuates immune suppression by RSV, resulting in resistance to S. pneumoniae. (A) Schedule of anti-AxI blocking $\mathrm{mAb}(5 \mu \mathrm{g} /$ dose, 4 doses) treatment for secondary bacterial infection after RSV infection. (B) Changes in the survival rate and (C) body weight of antiAxI mAb-treated or control (IgG-treated) mice infected with S. pneumoniae and/or RSV. (D) S. pneumoniae loads in BAL fluid from IgG- and anti-AxI mAb-treated mice infected with S. pneumoniae and/or RSV on day 1 after S. pneumoniae infection. (E) Representative H\&E-stained lung tissue sections from mice on day 1 after S. pneumoniae infection. Scale bars: $200 \mu \mathrm{m}$ (upper), $50 \mu \mathrm{m}$ (lower). (F) Numbers of inflammatory cells in BAL fluid from each treatment group on day 1 after S. pneumoniae infection. (G) IFN- $\gamma$ levels in BAL fluid from IgG- and anti-Axl mAb-treated mice on day 1 after S. pneumoniae infection. The data are expressed as the mean \pm SEM; $n=10$ (B and $\mathbf{C}$ ), $n=4-6$ (except for $\mathbf{B}$ and $\mathbf{C}$ ). Representative results from 2 independent experiments are shown. The following statistical tests were used: Gehan-Breslow-Wilcoxon test (A) and 1-way ANOVA (C, D, F, and $\mathbf{G}) .{ }^{*} P<0.05 ;{ }^{* *} P<0.01$.

IL-1 $\beta$ production and CXCL2-mediated neutrophil infiltration, which was associated with increased susceptibility to secondary pneumococcal infection (Figure 11).

Our findings demonstrated that Gas6/Axl-induced polarization of resident (MO-like) alveolar macrophages into M2-like macrophages during RSV infection is a key event to exacerbate pneumococcal pneumonia. The expression of Gas6 was initially detected in the alveolar epithelial cells on day 4 after infection. Subsequently, the macrophages exhibited enhanced Gas6 production by day 8 after infection. The kinetics of Gas 6 production suggested that macrophage polarization is triggered by epithelial cell-derived Gas6 during the early phase. From days 4 to 8 , the macrophages are polarized into M2-like macrophages, which also produce Gas6 via STAT6 activation $(16,38)$. During this period, 
A 8.5Gy $\gamma$-ray $\quad$ WT or Axt-1-

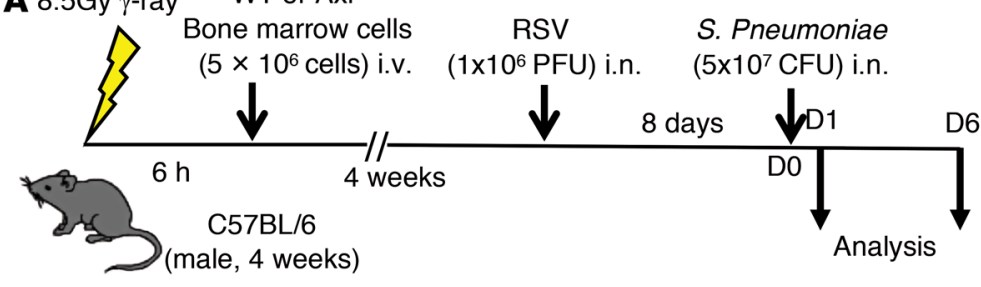

C

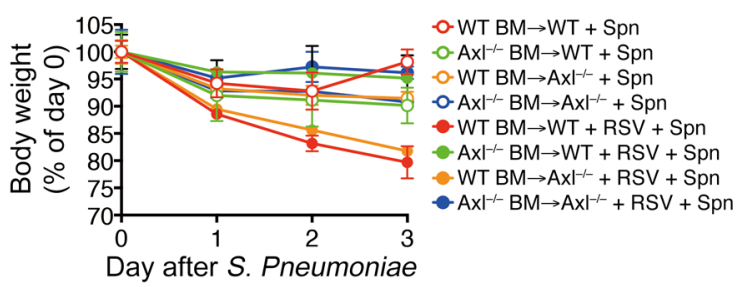

D S. pneumoniae

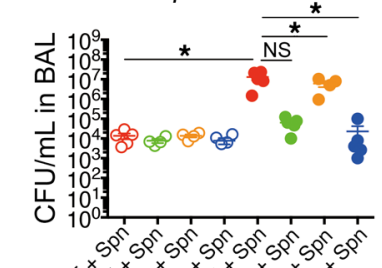

2056

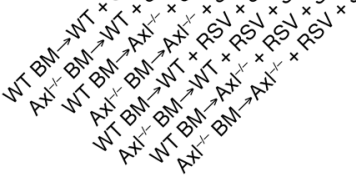

B

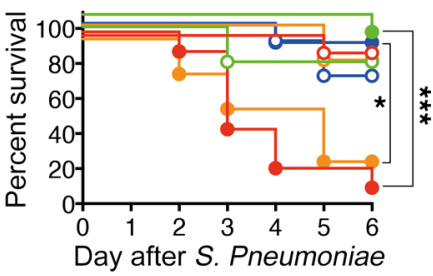

- $W T$ BM $\rightarrow$ WT + Spn

$\sim \mathrm{AxI}^{-1-} \mathrm{BM} \rightarrow \mathrm{WT}+\mathrm{Spn}$

- - WT BM $\rightarrow \mathrm{AxI}^{-1-}+\mathrm{Spn}$

○. $A x^{-1-} \mathrm{BM} \rightarrow \mathrm{Axl}^{-1-}+\mathrm{Spn}$

- WT BM $\rightarrow W T+R S V+S p n$

$-A x l^{-1-} B M \rightarrow W T+R S V+S p n$

- WT BM $\rightarrow \mathrm{Axl}^{-1-}+\mathrm{RSV}+\mathrm{Spn}$

- $\mathrm{AxI}^{-1-} \mathrm{BM} \rightarrow \mathrm{Axl}^{--}+\mathrm{RSV}+\mathrm{Spn}$
E

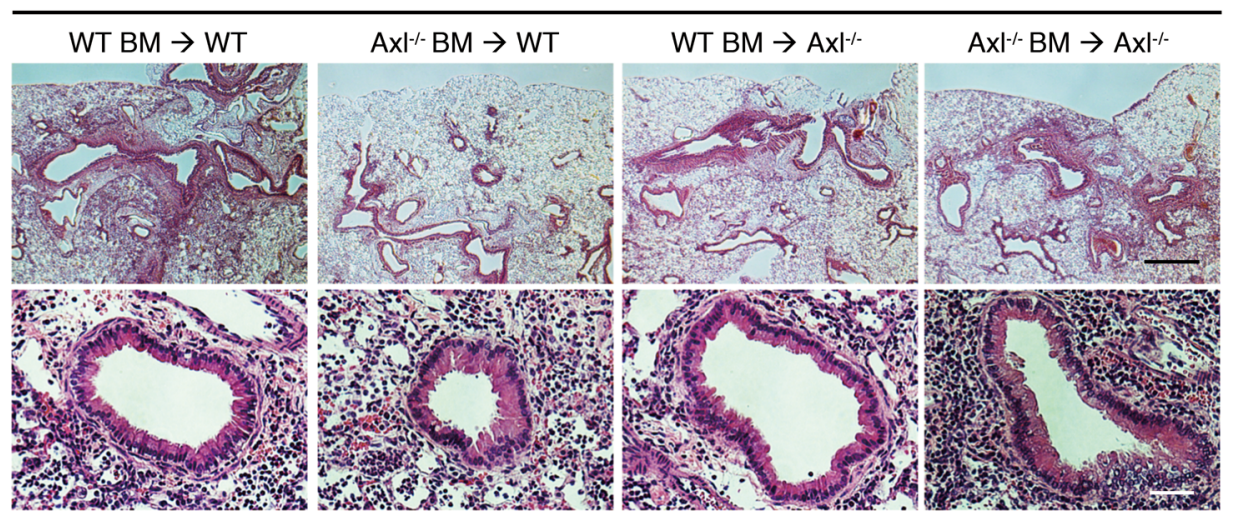

$\mathrm{RSV}+\mathrm{Spn}$

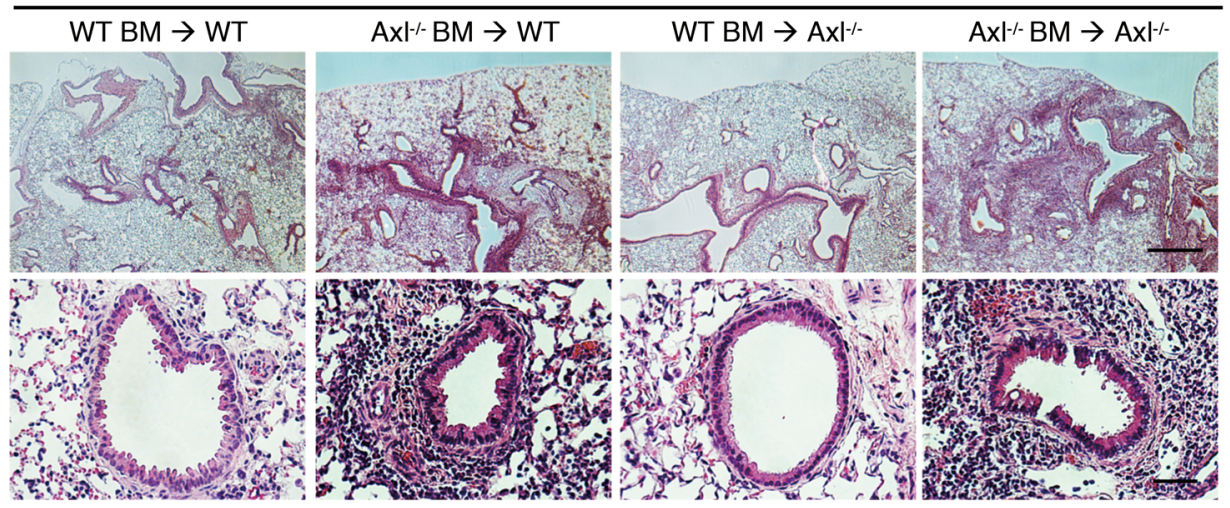

$\mathbf{F}$

Neutrophil

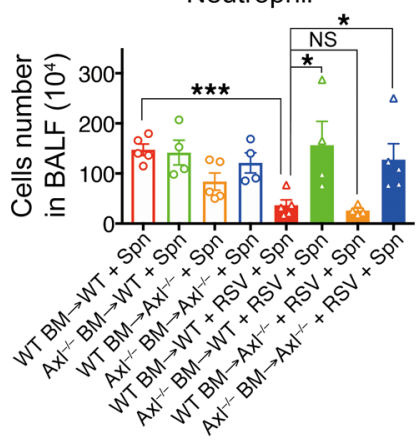

G

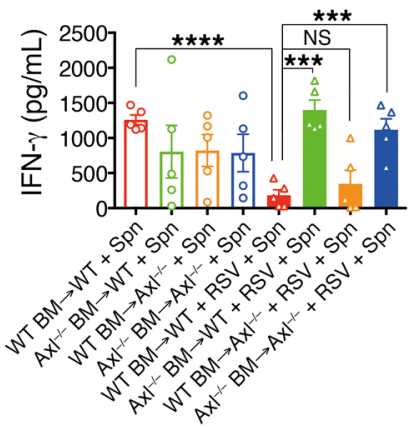

Figure 5. Axl on the alveolar macrophages increases susceptibility to S. pneumoniae infection after RSV infection. (A) To construct the bone marrow chimeric mice, the bone marrow (BM) cells of WT and $A x^{-1-}$ mice were adaptively transferred to WT and $A x l^{1-}$ mice. These mice were infected with S. pneumoniae at day 8 after RSV infection and monitored until day 6 after S. pneumoniae. (B) Change of survival rate, (C) body weight, and (D) titer of S. pneumoniae in the BAL fluid at day 6 after S. pneumoniae infection. (E) Representative H\&E-stained lung tissue sections on day 1 after $S$. pneumoniae infection. Scale bars: $200 \mu \mathrm{m}$ (upper), $50 \mu \mathrm{m}$ (lower). (F) Number of neutrophils in the BAL fluid on day 1 after S. pneumoniae infection. (G) The levels of IFN- $\gamma$ in the BAL fluid from mice on day 1 after $S$. pneumoniae infection. The data are expressed as the mean \pm SEM; $n=8-10$ (B and C), $n=4-6$ (except for B, C). Representative results from 2 independent experiments are shown. The following statistical tests were used: Gehan-Breslow-Wilcoxon test (B) and 1-way ANOVA (D, F, and $\mathbf{G}) .{ }^{*} P<0.05 ;{ }^{* *} P<0.01 ;{ }^{* *} P<0.001 ;{ }^{* * *} P<0.0001$. 
A

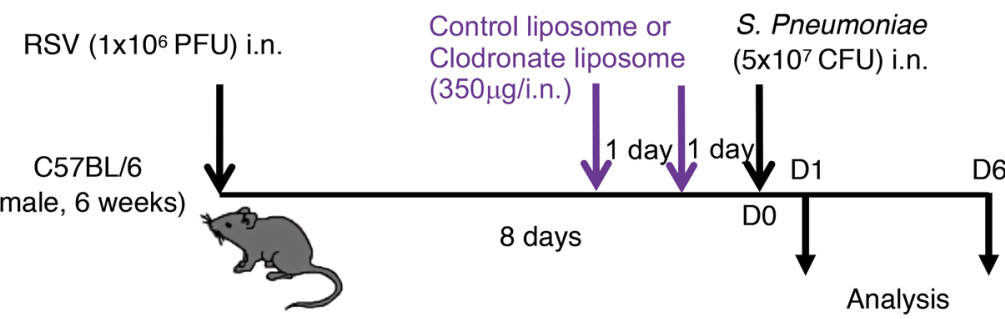

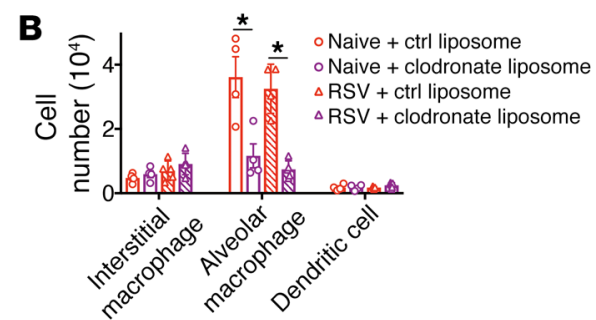

C

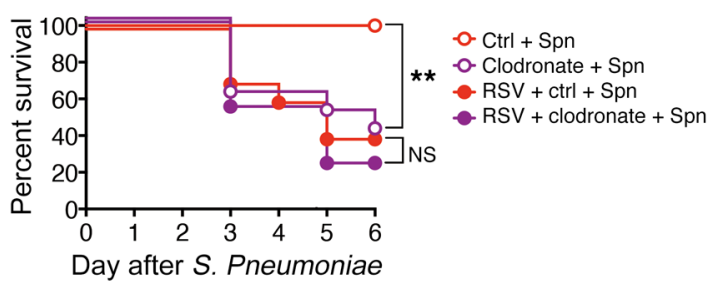

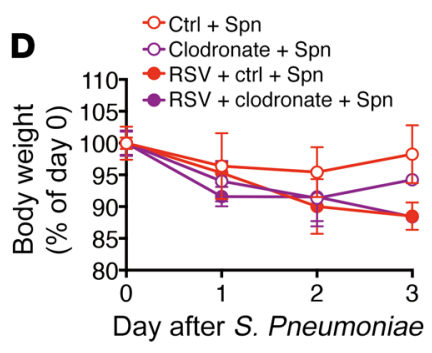

Day after $S$. Pneumoniae

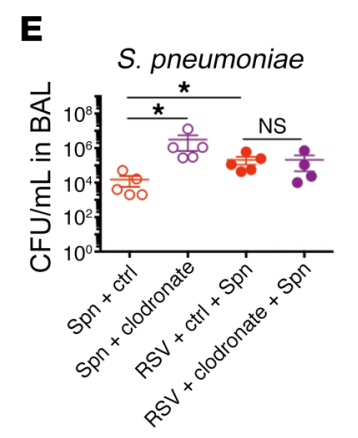

$\mathbf{F}$

$\mathrm{Ctrl}+\mathrm{Spn}$

Clodronate $+\mathrm{Spn}$

$\mathrm{RSV}+\mathrm{ctrl}+\mathrm{Spn}$

RSV + clodronate + Spn
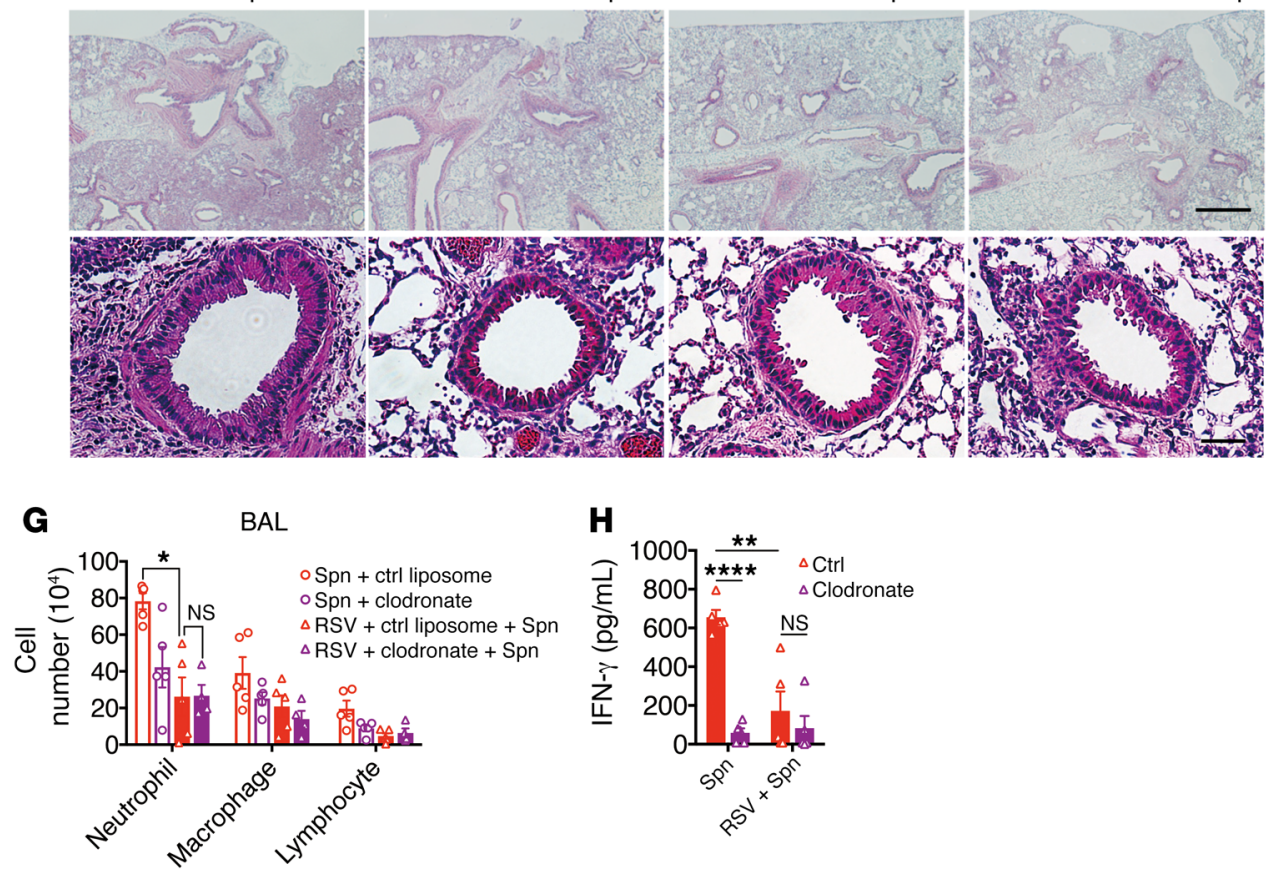

Figure 6. RSV infection suppresses the defensive response of alveolar macrophages to S. pneumoniae. (A) Kinetic analysis of clodronate liposome (clodronate; $350 \mu \mathrm{g} /$ dose, 2 doses) treatment for S. pneumoniae infection after RSV infection. (B) The number of interstitial and resident alveolar macrophages and dendritic cells in the lung of control liposome-treated and clodronate liposome-treated naive mice or mice on day 8 after RSV infection. (C) Changes in the survival rate and (D) body weight of clodronate-treated or control (liposome)-treated mice infected with S. pneumoniae. (E) S. pneumoniae loads in the BAL fluid from clodronate-treated mice infected with S. pneumoniae. (F) Representative H\&E-stained lung tissue sections from clodronate-treated mice on day 1 after S. pneumoniae infection. Scale bars: $200 \mu \mathrm{m}$ (upper), $50 \mu \mathrm{m}$ (lower). (C) Numbers of inflammatory cells in the BAL fluid from each treatment group on day 1 after S. pneumoniae infection. (H) The levels of IFN- $\gamma$ in the BAL fluid from each treatment group on day 1 after S. pneumoniae infection. The data are expressed as mean \pm SEM; $n=10$ (C and $\mathbf{D}), n=4-6$ (except for $\mathbf{C}$ and $\mathbf{D}$ ). Representative results from 2 independent experiments are shown. The following statistical tests were used: 1-way ANOVA (B, E, G, and $\mathbf{H})$ and Gehan-Breslow-Wilcoxon test $(\mathbf{C})$. ${ }^{*} P<0.05$; ${ }^{* *} P<0.01 ;{ }^{* * *} P<0.0001$.

the Gas6/Axl-triggered molecular cascades regulate macrophage polarization. Although the Gas6/Axl axis-mediated autophagy induction in macrophages ameliorates IL-18 production by inhibiting the NLRP3 inflammasome activation (39), further studies are needed to elucidate the exact mechanism underlying the regulation of phenotypic conversion of M0-like to M2-like macrophages by the Gas6/Axl axis after RSV infection.
The Gas6/Axl axis is required for the polarization of macrophages but is insufficient for the induction of M2-like macrophages. The polarization into M2-like macrophages additionally requires various Th 2 cytokines, such as IL- 4 and IL-13 induced by RSV infection (32). However, it is unlikely that the Th2 cells are the cellular source of these cytokines because previous studies have reported that the Th2 cells produced Th 2 cytokines in the BAL fluid 

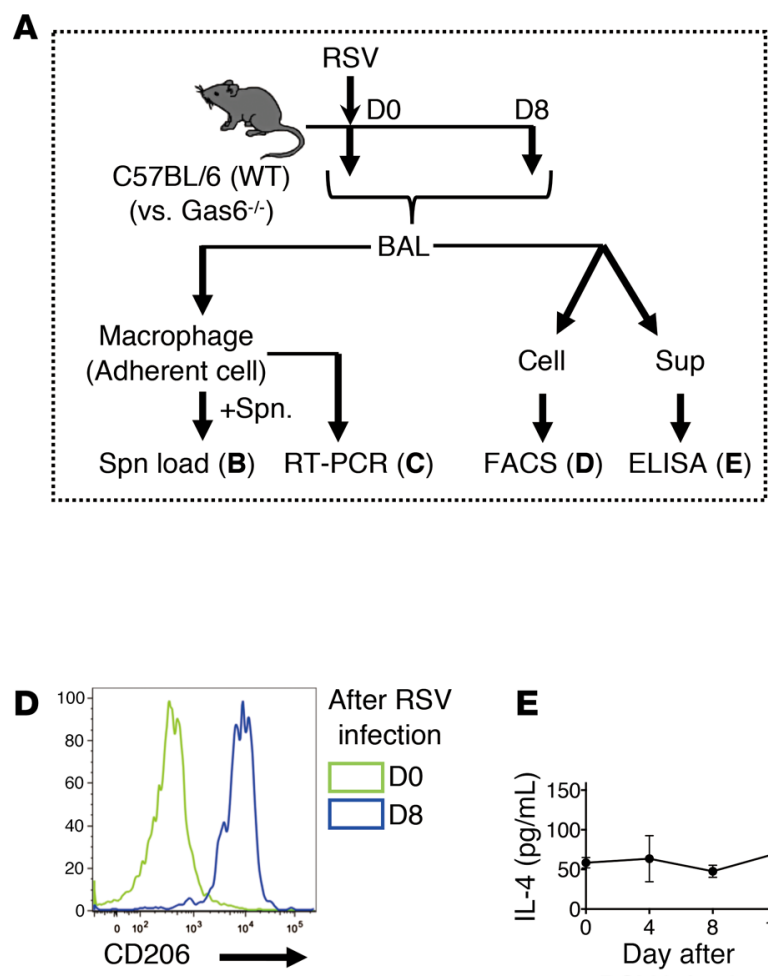

E
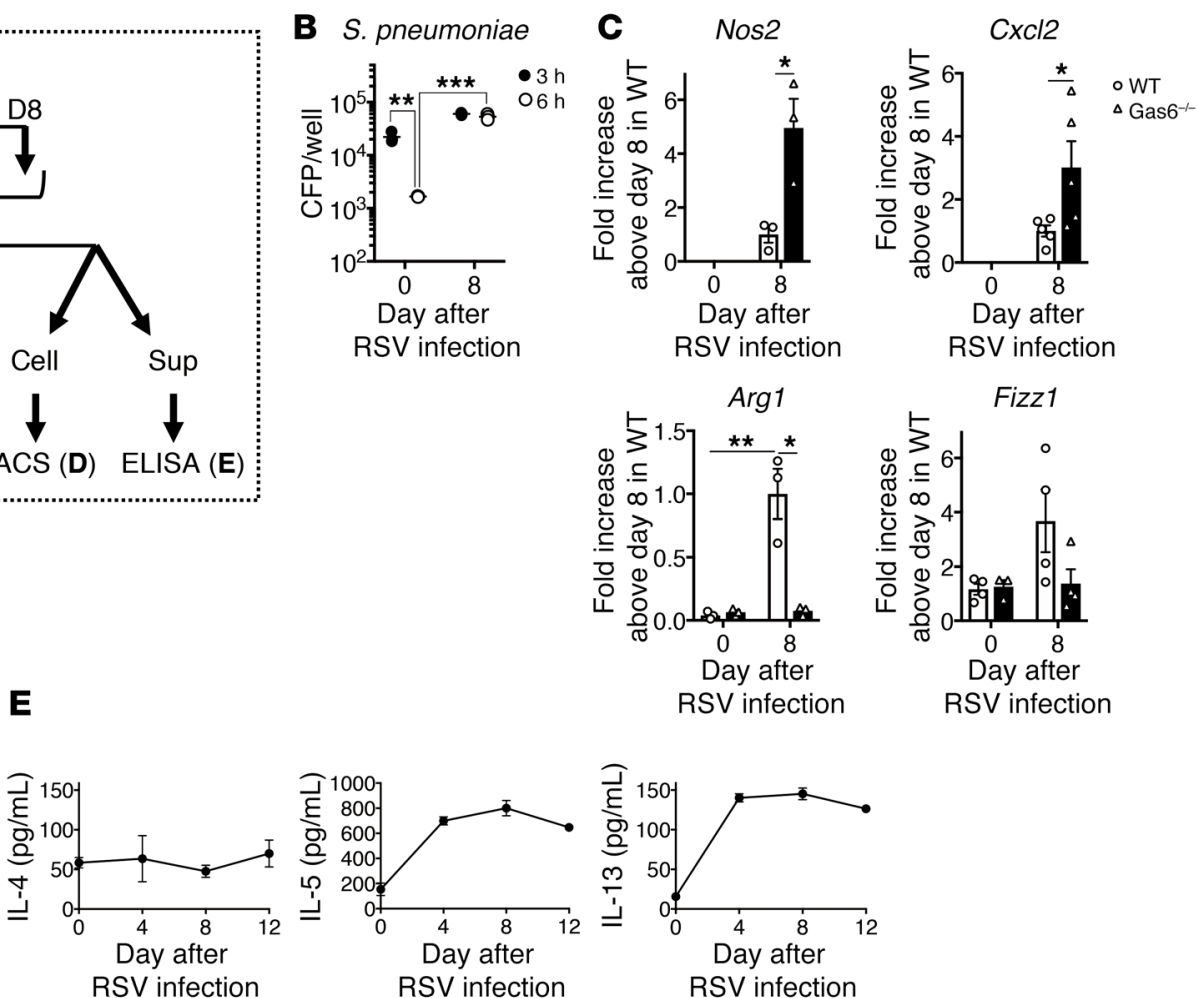

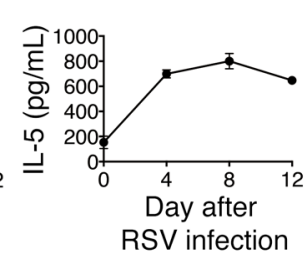

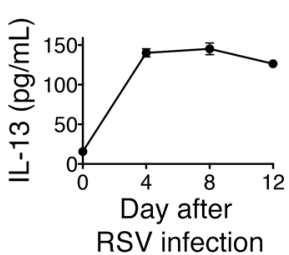

Figure 7. Gas6/Axl axis polarizes alveolar macrophages (M0-like) into M2-like phenotype that does not exhibit antibacterial activity. (A) Kinetic change of phenotype and antibacterial function of alveolar macrophages generated from naive mice and mice on days 4 and 8 after RSV infection. (B) Titers of S. pneumoniae after coculture of these alveolar macrophages with S. pneumoniae for 3 and 6 hours. (C) Quantitative real-time PCR analysis of Nos2, Cxc/2, Arg1, and Fizz1 in these alveolar macrophages. (D) Expression of CD206 by cells (CD11c $\left./ F 4 / 80^{+}\right)$in the BAL fluid from naive mice and mice on days 4 and 8 after RSV infection. (E) Temporal changes in the production of Th2 cytokines and phenotype of macrophages after RSV infection. The data are expressed as mean \pm SEM; $n=3-5$ (B-D), $n=5$ (E). Representative results from 2 independent experiments are shown. The following statistical tests were used: 1-way ANOVA (B and C). ${ }^{*} P<0.05,{ }^{* *} P<0.01,{ }^{* * *} P<0.001$.

during the late phase of RSV infection and not in the early phase (day 4) (40). The alternative sources of Th2 cytokines include group 2 innate lymphoid cells (ILC2) that produce the Th2 cytokines upon stimulation with IL-33, which is reported to be produced in the epithelial cells after RSV infection $(41,42)$. Similarly, we also observed the suppressive effects of Gas6 on IFN- $\gamma$ production in the early phase of RSV infection (16). Therefore, Gas6-mediated M2-like polarization may be regulated indirectly through the upregulation of ILC2-derived Th2 cytokines or suppression of Th1 cytokines. However, the possibility that Gas6 directly modulates MO-like to M2-like polarization cannot be excluded. Hence, it is crucial to elucidate the mechanism by which Gas6 mediates macrophage polarization during RSV infection on a molecular basis.

The role of Gas6/Axl-mediated regulation in the non-RSV pathogen infections must be elucidated. The Gas6 protein is upregulated after infection by other pathogens in the murine models of fungal asthma and invasive pulmonary aspergillosis. In addition to the enhanced expression after RSV infection, Gas6 and Axl are highly expressed in the immune cells and airway epithelial cells after fungal infection $(17,25,43)$. However, there are limited clinical studies linking enhanced Gas6 and Axl expression with fungal and bacterial infections (44). The initial fungal infection may regulate antibacterial immunity through a similar Gas6/ Axl-mediated pathway. The initial influenza infection is reported to be frequently associated with subsequent pneumococcal infection. The following mechanisms are suggested to account for the exacerbated pneumococcal infection: IL-10-mediated suppression of invariant NKT activation (45), desensitization of TLR signals (46), type I IFN-mediated inhibition of CXCL1 and CXCL2 production (47), and virus-induced downregulation of the type A scavenger receptor MARCO (48). However, Gas6 expression was not detected after H1N1 influenza virus infection when the initial influenza virus infection led to increased susceptibility to secondary pneumococcus infection in mice (16). Thus, it is highly likely that the cascades involved in influenza-associated pneumococcus infection are different from those involved in RSV infections. The expression levels of Gas6 after infection are largely determined by the antigenic properties of individual pathogens. For RSV, G glycoprotein is reported to bind CX3CR1 (49), which initiates Gas6 production (50). Further studies are required for dissecting the mechanisms underlying infection-induced Gas6 production and immune cell polarization.

In conclusion, we elucidated the cellular and molecular mechanisms that mediate increased susceptibility to $S$. pneumoniae infection after RSV infection in a mouse model. Future studies using a large number of clinical samples from patients with RSV infection are needed to assess whether the results obtained in the mouse model can be extrapolated to the clinical samples. 
A

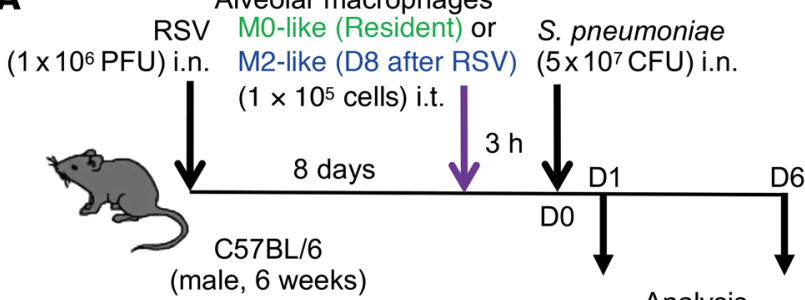

B

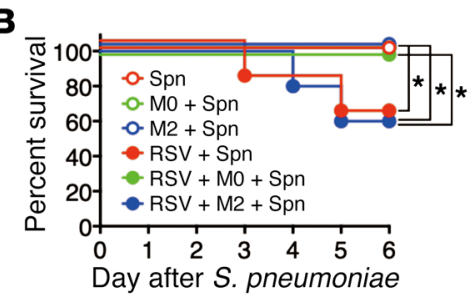

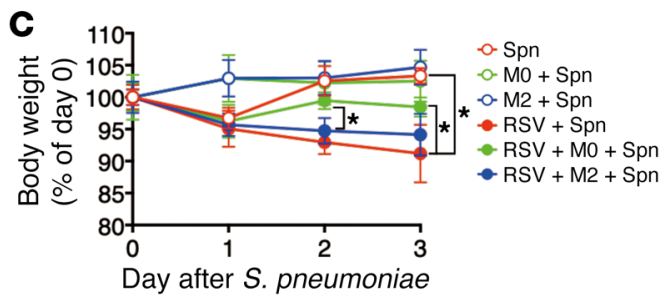

Day after $S$. pneumoniae

Analysis

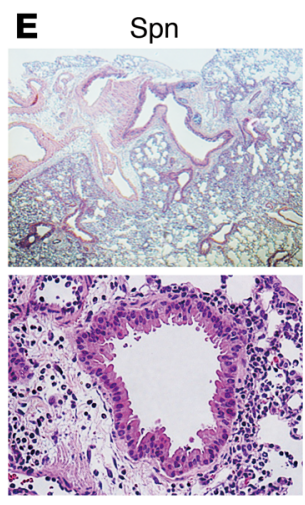

$\mathrm{RSV}+\mathrm{Spn}$

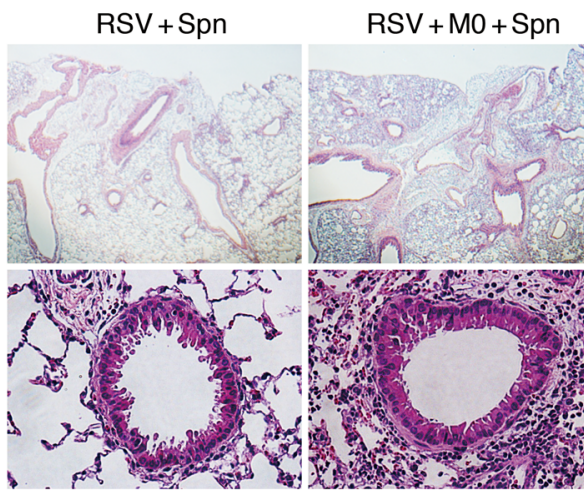

$\mathrm{MO}+\mathrm{Spn}$

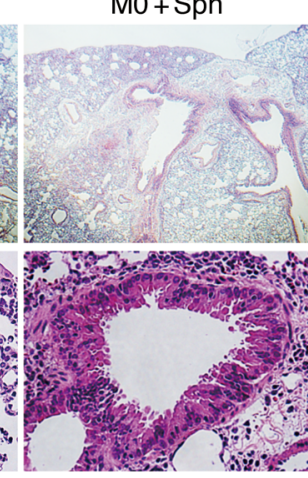

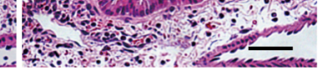

$\mathrm{RSV}+\mathrm{M} 2+\mathrm{Spn}$

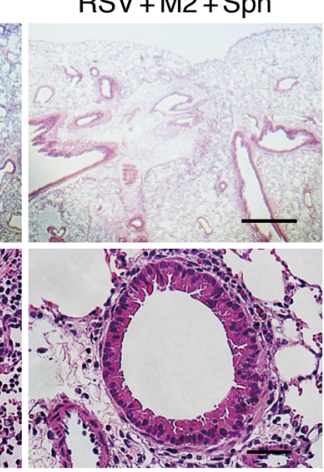

$\mathbf{F}$

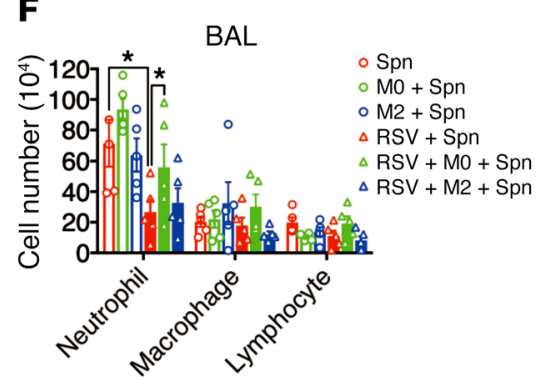

G

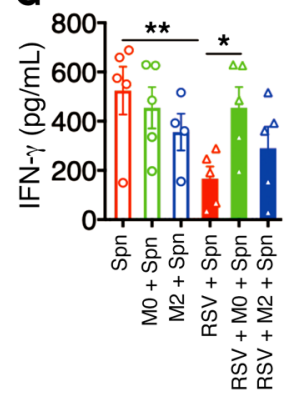

Figure 8. Transfer of resident alveolar macrophages protected the mice against secondary bacterial infection. (A) Kinetic schedule of resident (MO-like) and M2-like macrophage $\left(1 \times 10^{5}\right.$ cells) transfer via intratracheal (i.t.) injection during secondary bacterial infection after RSV infection. (B) Changes in the survival rate, (C) body weight, and (D) S. pneumoniae loads in the BAL fluid of mice infected with S. pneumoniae after RSV infection and after injection with MO-like and M2-like macrophages. (E) Representative H\&E-stained lung tissue sections from MO-like and M2-like macrophage-transferred mice on day 1 after S. pneumoniae infection. Scale bars: $200 \mu \mathrm{m}$ (upper), $50 \mu \mathrm{m}$ (lower). (F) Numbers of inflammatory cells in the BAL fluid from each treatment group on day 1 after $S$. pneumoniae infection. (C) The levels of IFN- $\gamma$ in the BAL fluid from each treatment group on day 1 after $S$. pneumoniae infection. The data are expressed as mean \pm SEM; $n=10$ (B, C), $n=5-6$ (except for $\mathbf{B}$ and $\mathbf{C}$ ). Representative results from 2 independent experiments are shown. The following statistical tests were used: Gehan-Breslow-Wilcoxon test (B) and 1-way ANOVA (C, D, F, and $\mathbf{C}) .{ }^{*} P<0.05 ;{ }^{*} P<0.01$.

Our study also identified the Gas6/Axl axis as a potentially novel therapeutic target for preventing severe secondary $S$. pneumoniae infection. However, it should be noted that manipulation of the Gas6/Axl axis may increase the risk of excessive inflammation due to the production of antibacterial mediators. It is important to evaluate the therapeutic effect and the associated side effects of the Axl inhibitor for restoring antibacterial immunity attenuated by prior RSV infection.

\section{Methods}

Mice. The male mice were maintained in a specific pathogen-free facility at the National Institute of Infectious Diseases (NIID, Tokyo, Japan). 
A

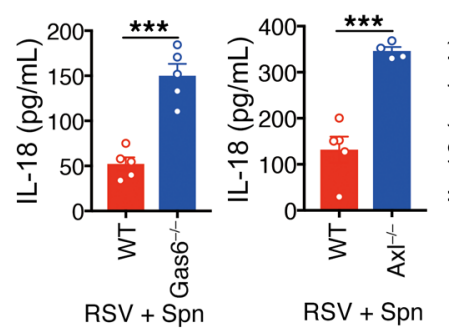

B

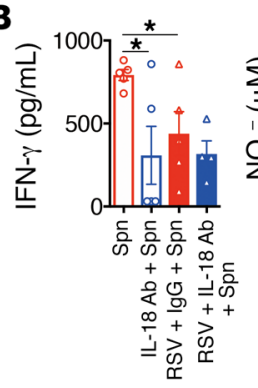

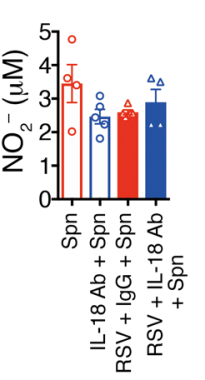

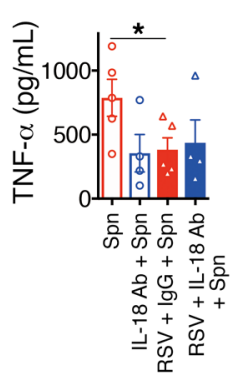

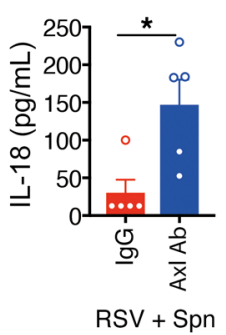

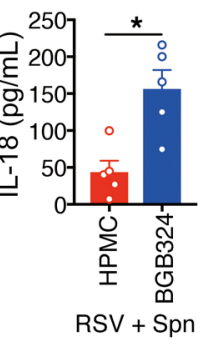

C

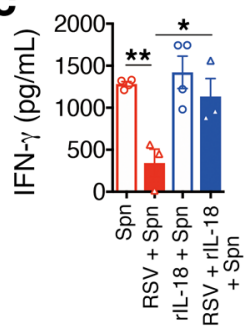

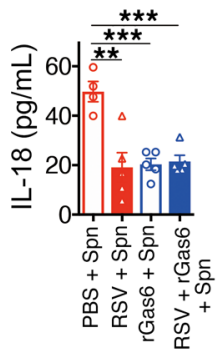

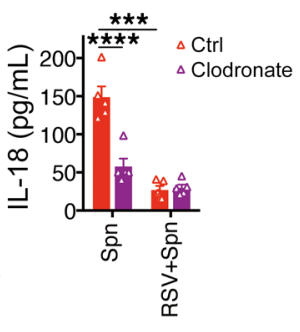

D

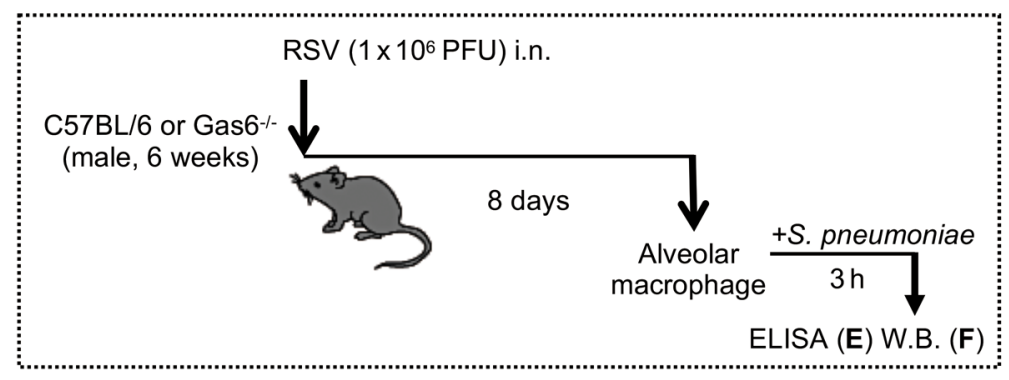

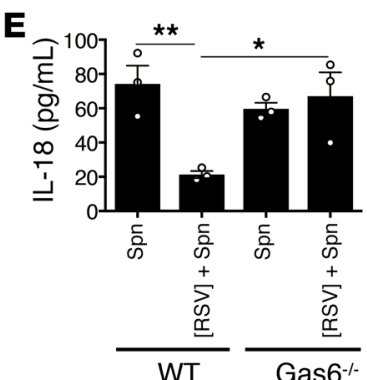

F Macrophage
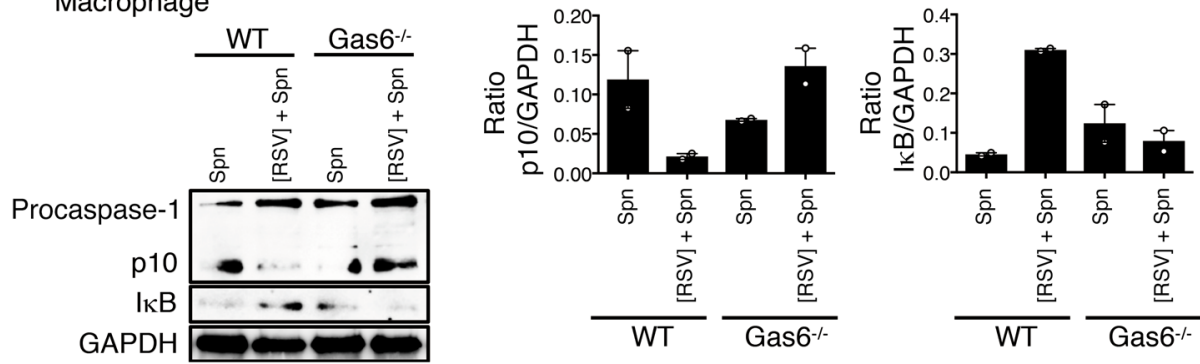

Figure 9. Gas6/Axl axis suppresses IL-18 production from macrophages by suppressing the activation of caspase-1 and NF- $\mathrm{KB}$. (A) The levels of IL-18

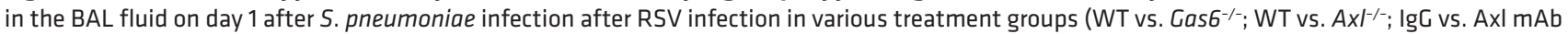
treatment; hydroxypropyl methylcellulose vs. BGB324 treatment; PBS vs. rGas6 treatment; and control liposome vs. clodronate liposome treatment). The levels of IFN- $\gamma$, NO, and TNF- $\alpha$ in the BAL fluid from each group at day 1 after S. pneumoniae infection in the anti-IL-18 mAb-treated (B) and recombinant IL-18-treated (C) mice infected with S. pneumoniae and/or RSV. ( $D$ and E) IL-18 production in the alveolar macrophages isolated from WT and Gas6 ${ }^{-/-}$mice on day 8 after RSV infection and stimulated with S. pneumoniae for 1 hour. (F) Activation of caspase-1 and degradation of I $\mathrm{kB}$ in the alveolar macrophages isolated from WT and $\mathrm{Cas6}^{-1-}$ mice on day 8 after RSV infection and stimulated with S. pneumoniae for 3 hours (left panel). The ratios of cleaved caspase-1 (p10) and the IKB to GAPDH ratio are shown (right panel). The data are expressed as mean \pm SEM; $n=4-6$ ( $\mathbf{A}$ and $\mathbf{B}), n=2-3$ (E and $\mathbf{F})$. Representative results from 2 independent experiments are shown. The following statistical tests were used: 2 -tailed Student's $t$-test (A, for WT vs. Gas6 $6^{-1-}$; WT vs. Axl ${ }^{-1-}$; IgG vs. AxI mAb treatment; hydroxypropyl methylcellulose vs. BGB324 treatment), and 1-way ANOVA (A, for PBS vs. rGas6 treatment; and control liposome vs. clodronate liposome treatment, B, C, and E). ${ }^{*} P<0.05 ;{ }^{* *} P<0.01 ;{ }^{* * *} P<0.001$; ${ }^{* * * *} P<0.0001$.

The C57BL/6J (WT) mice (6 weeks old) were obtained from Japan SLC, Inc. The $\mathrm{Gas}^{-/-}$mice were a gift from Russell Taichman (University of Michigan, Ann Arbor, Michigan, USA). The $A x^{t^{--}}$mice were a gift from Carla Rothlin (Yale University, New Haven, Connecticut, USA). All $\mathrm{Gas6}^{-/-}$and $\mathrm{Axl}^{-/-}$mice used in this study were backcrossed to C57BL/6 J background for 10 or more generations. These mice were subsequently bred and maintained in a specific pathogen-free colony at NIID.
Mouse model of secondary bacterial infection after RSV infection. The RSV A2 strain was originally provided by Stokes Peeble (Vanderbilt University, Nashville, Tennessee, USA) and propagated in the cultured HEp-2 cells (American Type Culture Collection). The S. pneumoniae D39 strain was provided by Shigeki Nakamura at NIID and was maintained on $10 \%$ (vol/vol) sheep blood agar plates (BD Biosciences) overnight at $37^{\circ} \mathrm{C}$ and cultured in $10 \mathrm{~mL}$ of trypticase soy broth for approximately 4 
A
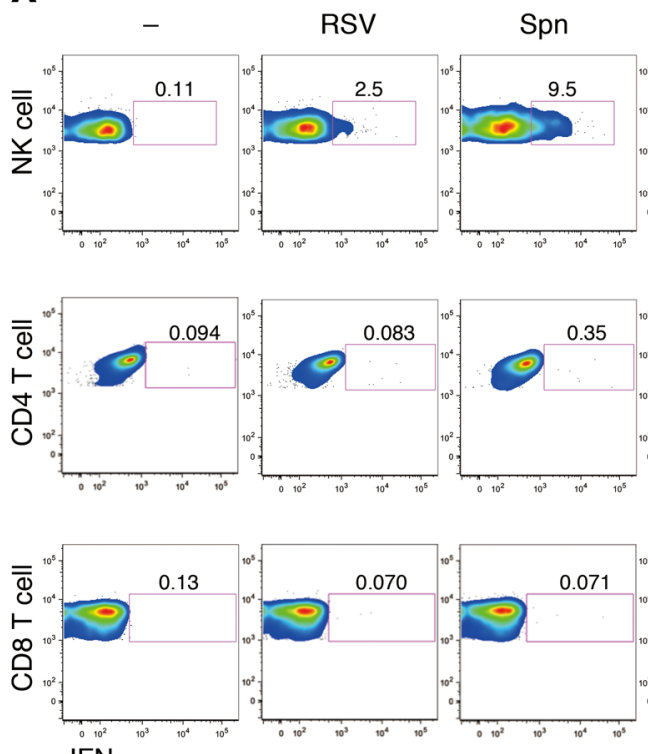

IFN- $\gamma$
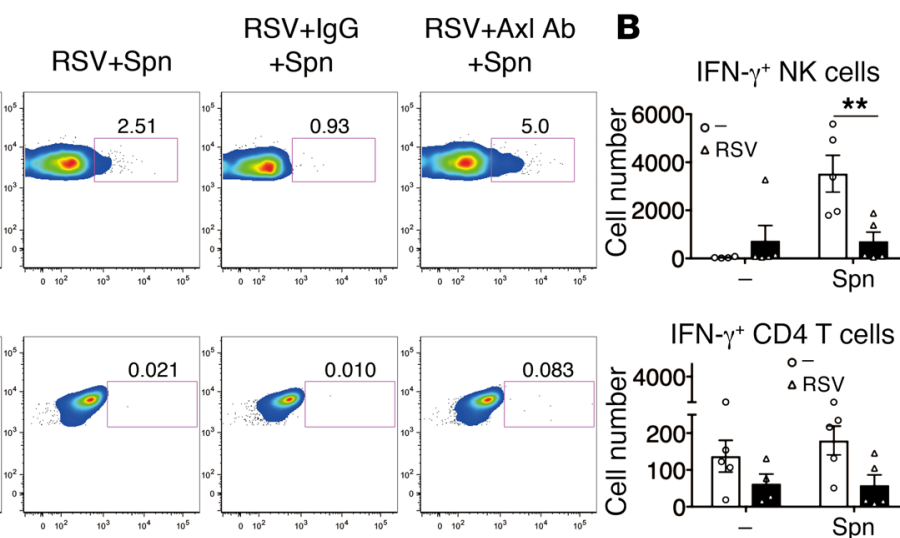

C

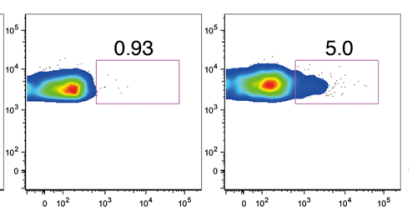

IFN- $\gamma^{+}$CD4 T cells

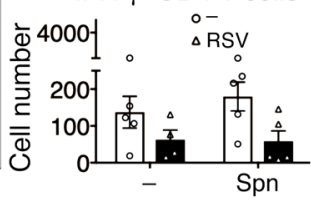

IFN- $\gamma^{+}$CD8 T cells

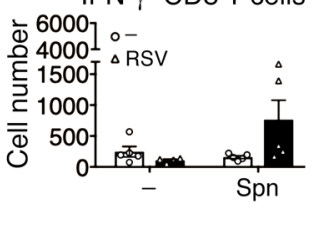

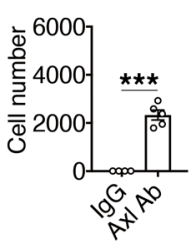
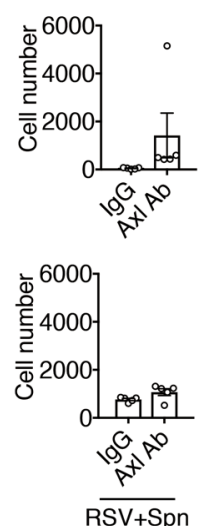

Figure 10. RSV infection-induced Gas6/AxI axis suppresses IFN- $\gamma$ production in NK cells after S. pneumoniae infection. (A) Representative flow cytometry analysis of IFN- $\gamma$ production by NK cells, CD4 ${ }^{+} \mathrm{T}$ cells, and CD8 ${ }^{+} \mathrm{T}$ cells during secondary bacterial infection after RSV infection in the control IgG-treated or anti-AxI mAb-treated mice. (B) Numbers of cells positive for IFN- $\gamma$ in the lungs of control mice and (C) AxI mAb-treated mice on day 1 after $S$. pneumoniae infection. The data are expressed as mean \pm SEM; $n=4-5$. Representative results from 2 independent experiments are shown. The following statistical tests were used: One-way ANOVA (B), 2-tailed Student's $t$ test (C). ${ }^{* *} P<0.01$; ${ }^{* *} P<0.001$.

hours at $37^{\circ} \mathrm{C}$ (until mid-log phase; $\mathrm{OD}_{620 \mathrm{~nm}}=0.4$ ). As shown in Supplemental Figure 2A, the C57BL/6J mice were injected (i.n.) with RSV $\left(10^{6}\right.$ $\mathrm{PFU}$ ). Eight days later, the mice were administered (i.n.) S. pneumoniae $\left(5 \times 10^{7} \mathrm{CFU} / \mathrm{mouse}\right)$. On days $1,2,3$, and 6 after $S$. pneumoniae infection, the mice were anesthetized with isoflurane, and the whole-lung lobes from mice were dissected and snap-frozen for protein, cellular, and transcript analyses or fixed in $10 \%$ formalin for histological analysis.

In vivo treatment with recombinant proteins, antibodies, or inhibitor. In the mouse model of secondary bacterial infection after RSV infection, the animals were treated with several agents. For treatment 1 , starting on day 4 after RSV infection, the mice received only PBS or rGas6 (mouse protein; 3 doses; $1 \mu \mathrm{g} /$ dose; R\&D Systems) every other day until S. pneumoniae infection. For treatment 2, mice were injected (i.p.) with human IgG1 (control group; $5 \mu \mathrm{g} /$ dose; Genetech) or antiAxl mAb (monoclonal anti-human Axl mAb YW327.6S2; $5 \mu \mathrm{g} /$ dose; Genetech). For treatment 3, the mice were treated with BGB324 (250 $\mu \mathrm{g} /$ dose; BerGenBio) or hydroxypropyl methylcellulose (Alfa Aesar). For treatment 4, the mice were injected (i.p.) with mouse IgG2a (control group; $300 \mu \mathrm{g} /$ dose; Bio X Cell) or anti-NK1.1 mAb (monoclonal anti-mouse NK1.1 mAb, PK136; $200 \mu \mathrm{g} /$ dose; Bio X Cell). For treatment 5, the mice were injected (i.p.) with mouse IgG2a (control group; $200 \mu \mathrm{g} /$ dose; Bio X Cell) or anti-IL-18 mAb (monoclonal anti-mouse NK1.1 mAb; YIGIF74-1G7; $200 \mu \mathrm{g}$ /dose; Bio X Cell). For treatment 6, the mice were injected (i.p.) with PBS (control) or rIL-18 (mouse protein; 2 doses; $1 \mu \mathrm{g}$ /dose; Medical \& Biological Laboratories Co. LTD) every other day until S. pneumoniae infection. For treatment 7 , the mice were injected (i.p.) with mouse IgG2a $(250 \mu \mathrm{g} /$ dose; Bio X Cell) or anti-mouse Ly6G mAb; BP0075-1; $250 \mu \mathrm{g}$ /dose; Bio X Cell). Detailed treatment timings are specified in the respective figures.
Generation of bone marrow chimera in mice. For generation of bone marrow chimeric mice, the recipient WT and $\mathrm{Axl}^{-/-}$mice or Gas6 $6^{-/-}$ mice ( 5 weeks old) were lethally irradiated with 8.5 Gy of gamma ray before transplantation with bone marrow cells $\left(5 \times 10^{6}\right)$ from 5 -weekold donor male WT and $\mathrm{Axl}^{-/-}$or $\mathrm{Gas}^{-/-}$mice via tail vein injection. After a 4-week recovery period, the mice were infected with RSV followed by $S$. pneumoniae infection.

Clodronate liposome treatment of mice after RSV infection before secondary bacterial infection. To deplete the alveolar macrophages, $30 \mu \mathrm{L}$ of clodronate liposome ( $350 \mu$ g; FormuMax Scientific Inc.) or $30 \mu \mathrm{L}$ of control liposome suspension (FormuMax Scientific Inc.) was administered (i.n.) to mice on days 6 and 7 after RSV infection. These mice were infected with $S$. pneumoniae on day 8 after RSV infection.

Adoptive macrophage transfer. The M0-like and M2-like macrophages were obtained from naive and RSV-infected mice on day 8 after RSV infection, respectively, by BAL. Next, $1 \times 10^{5}$ macrophages were adoptively transferred by injection (i.t.) into the RSV-infected mice. The mice were infected with $S$. pneumoniae at 3 hours after macrophage transfer.

BAL fluid collection and preparation of alveolar macrophages. The BAL fluid was collected from the naive and RSV-infected and/or $S$. pneumoniae-infected mice using a tracheal cannula with instillation of $1 \mathrm{~mL}$ of PBS. To obtain the macrophages from the BAL fluid, the cells $\left(4 \times 10^{5}\right.$ cells/well) were incubated in a 48 -well plate for 1 hour at $37^{\circ} \mathrm{C}$. The nonadherent cells were removed and washed 3 times with warm PBS. The resultant adherent cells included $96.4 \% \pm 2.4 \%$ of macrophages, which were analyzed by H\&E staining.

Detection of Gas6 production by the MO-like and M2-like alveolar macrophages ex vivo. The alveolar macrophages obtained from naive 


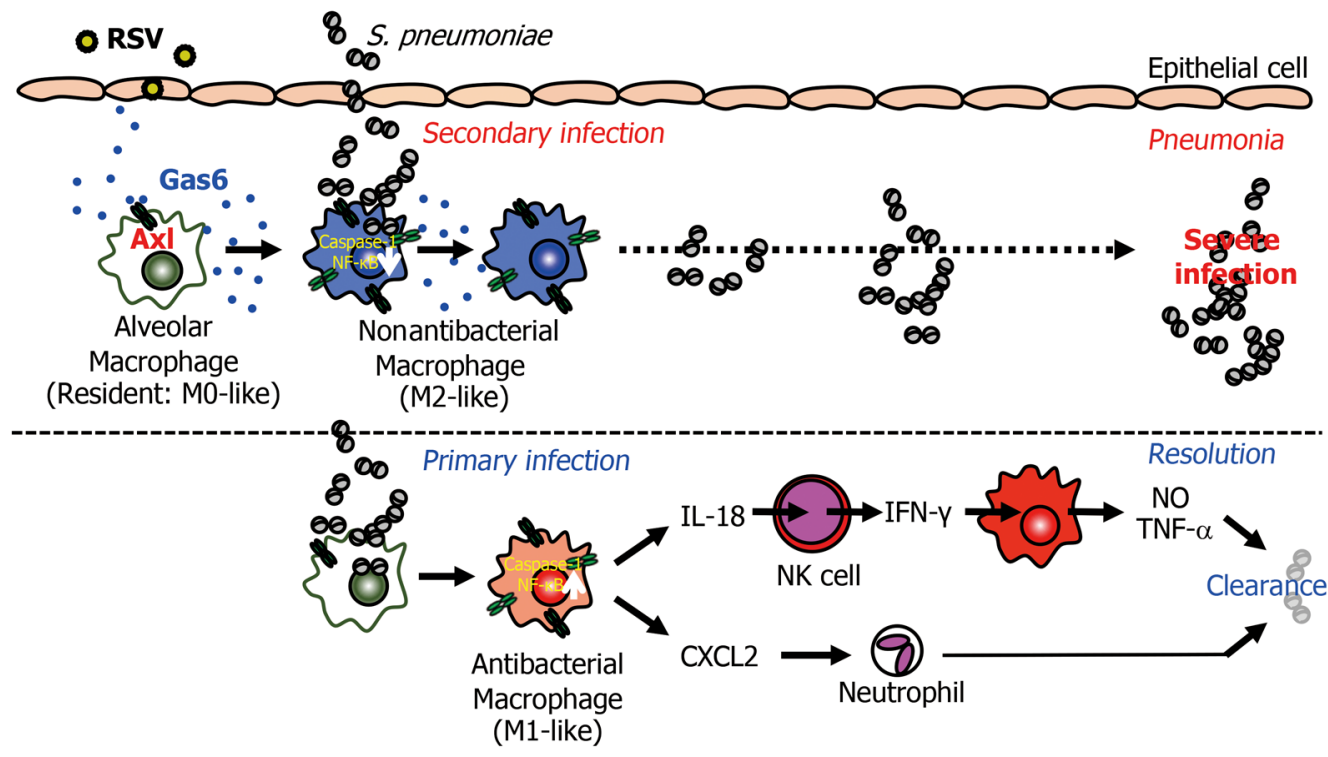

Figure 11. RSV-induced Gas6/AxI axis enhances susceptibility to bacterial infection. Shortly after RSV infection, the epithelial cells and alveolar macrophages produce Gas6. The Gas6/Axl axis polarizes antibacterial (MO-like) macrophages into nonantibacterial (M2-like) macrophages that inhibit the activation of caspase-1/IL-18. The low levels of IL-18/IFN- $\gamma$ in the macrophages and NK cells result in severe secondary pneumococcal infection.

and RSV-infected mice were incubated in a 48 -well plate for 1 hour at $37^{\circ} \mathrm{C}$. The supernatants were collected to determine the Gas 6 levels by ELISA. The cells were collected and the levels of Nos2, Tnf, Cxcl2, Arg1, and Fizz1 were determined by real-time PCR.

S. pneumoniae colony-forming assay. At the designated time points after infection, the BAL fluid was collected with $1 \mathrm{~mL}$ of PBS. The CFU of $S$. pneumoniae in the BAL fluid was enumerated by plating the serially diluted samples (in PBS) on blood agar and incubated at $37^{\circ} \mathrm{C}$ for 18 hours.

Determination of cytokine levels. The levels of Gas6, IFN- $\gamma$, TNF- $\alpha$, IL-1 $\beta$, IL-4, IL-5, IL-13, CXCL1, CXCL2, and leukotriene (LTB4) in the BAL fluid in vitro and ex vivo were determined using a standardized sandwich ELISA (R\&D Systems), following the manufacturer's instructions. The recombinant murine proteins (R\&D Systems) were used to generate the standard curves. The ELISA detection limits for the cytokines were as follows: Gas6, greater than $39.1 \mathrm{pg} / \mathrm{mL}$; IFN- $\gamma, 15.6 \mathrm{pg} /$ $\mathrm{mL}$; TNF- $\alpha, 15.6$ pg/mL; IL-1 $\beta, 7.8$ pg/mL; IL-4, 15.6 pg/mL; IL-5, 31.3 $\mathrm{pg} / \mathrm{mL}$; IL-13, $62.5 \mathrm{pg} / \mathrm{mL}$; CXCL1, 15.6 pg/mL; CXCL2, $15.6 \mathrm{pg} / \mathrm{mL}$; and LTB4, $10.3 \mathrm{pg} / \mathrm{mL}$. The mouse IL-18 level in the BAL fluid and supernatant of alveolar macrophages ex vivo were determined using standardized sandwich ELISA (Medical \& Biological Laboratories Co. LTD), following the manufacturer's instructions. The ELISA detection limit was consistently more than $12.5 \mathrm{pg} / \mathrm{mL}$. The levels of human Gas6 and IFN- $\gamma$ in patient supernatants were determined using the Duoset ELISA development kit (R\&D Systems), following the manufacturer's instructions. The ELISA detection limits for Gas6 and IFN- $\gamma$ were consistently greater than $15.6 \mathrm{pg} / \mathrm{mL}$ and $9.38 \mathrm{pg} / \mathrm{mL}$, respectively. All reactions were stopped by adding $25 \mu \mathrm{L}$ of $1 \mathrm{M} \mathrm{H}_{2} \mathrm{SO}_{4}$. The sample absorbance was measured at $450 \mathrm{~nm}$ using a Model 680 microplate reader (Bio-Rad).

NO determination. The concentration of NO was quantified in terms of nitrite concentration by the Griess reaction using an assay kit (DOJINDO), following the manufacturer's instructions. The absorbance at 540 $\mathrm{nm}$ was determined with a Model 680 microplate reader (Bio-Rad).
Whole-lung immunohistochemical and histological analyses. The whole lungs were fully inflated with $10 \%$ formalin, dissected, and placed in fresh $10 \%$ formalin at room temperature for 24 hours. Routine histological techniques were used to embed the tissue in paraffin. The immunohistochemical analysis was performed on $5-\mu \mathrm{m}$ wholelung sections. Briefly, the $5-\mu \mathrm{m}$ histological sections were dewaxed in xylene and rehydrated in graded ethanol series. The specific proteins were detected using the rabbit anti-mouse Gas6 polyclonal Ab (Santa Cruz Biotechnology; SC22759, 1:100), rabbit anti-mouse Axl polyclonal Ab (LifeSpan BioSciences; LS-B7213, 1:500), and rabbit anti-mouse uteroglobin Ab (Bioss Antibodies; bs-1487R, 1:100). Other tissue sections were incubated with the control IgG isotype $\mathrm{Ab}$. The slides were developed using the mouse HRP-(3,3'-diaminobenzidine) (DAB) cell- and tissue-staining kit (R\&D Systems), following the manufacturer's instructions. Additionally, the tissue sections were subjected to H\&E staining. The slides were visualized under a light microscope (Nikon TE2000), and the images were captured using a camera attached to the microscope.

Flow cytometry analysis. The whole-lung samples were obtained from all groups of mice on day 8 after RSV challenge and were incubated with collagenase type I $(1 \mathrm{mg} / \mathrm{mL}$; Thermo Fisher Scientific) and DNAse I ( $1 \mu \mathrm{g} / \mathrm{mL}$; Sigma-Aldrich) at $37^{\circ} \mathrm{C}$ for 45 minutes. The whole-lung cell suspensions were then incubated with the anti-CD16/ CD32 (2.4G2, BD Pharmingen; 553142, 1:200) antibodies, followed by incubation with the fluorescent dye-mAb conjugates: anti-CD45 (BD Pharmingen; 560694, 1:200), anti-CD3 (eBioscience; 17-5892-81, 1:500), anti-CD4 (BD Pharmingen; 550954,1:200), anti-CD8 (eBioscience; 11-0081-82, 1:500), anti-NK1.1 (Abcam; ab210337, 1:200), antiCD11b (BD Pharmingen; 562127, 1:500), anti-F4/80 (BD Pharmingen; 562127, 1:200), anti-CD11c (BD Pharmingen; 553801, 1:500), antiCD64 (BD Pharmingen; BioLegend, 139307, 1:200), anti-Siglec-F (BD Pharmingen; 562068, 1:200), anti-MHC II (BD Pharmingen; 557000, 1:333), anti-Ly6G (BioLegend, 141703, 1:160), anti-CD326 (BioLegend, 
118207, 1:200), anti-CD206 (BioLegend, 141703, 1:100), and anti-Axl (YW327.6S2; Genetech; conjugated to allophycocyanin using a labeling kit for 15 minutes). For intracellular staining of the cytokines, the lung cells $\left(10^{6}\right.$ cells per well) were stimulated with phorbol myristate acetate $(50 \mathrm{ng} / \mathrm{mL})$ and ionomycin $(1 \mu \mathrm{M})$ in the presence of GolgiStop (BD Cytofix/Cytoperm kit; BD Biosciences Pharmingen) at $37^{\circ} \mathrm{C}$ for 5 hours. The cells were resuspended in the fixation/permeabilization solution (BD Cytofix/Cytoperm kit; BD Pharmingen) and stained with the anti-IFN- $\gamma$ antibody (BioLegend, CA; 17-7041-81, 1:100) for 30 minutes. The data were acquired using a FACSCanto II machine and FACS Diva software 8.0 (BD Pharmingen). All data were analyzed using the FlowJo software package (Tree Star).

Western blotting. The lung homogenates and alveolar macrophages isolated from RSV-infected mice were cultured with $S$. pneumoniae for 3 hours. The samples were lysed using a lysis buffer. The lysates were then resolved by SDS-PAGE. The resolved proteins were transferred to a PVDF membrane using iBlot (Thermo Fisher Scientific). The membrane was then incubated with $5 \%$ skimmed milk prepared in Tris-buffered saline containing $0.1 \%$ Tween-20 at room temperature for 1 hour. The membrane was incubated with the rabbit anti-ІкB (Abcam, ab32518, 1:500), anti-caspase-1 (Santa Cruz Biotechnology, sc56036, 1:100), or anti-GAPDH (control) antibodies (Abcam, ab8245, 1:1000) for 1 hour at room temperature. The membrane was washed and incubated with the HRP-conjugated goat anti-rabbit antiIgG (Abcam, ab6721, 1:2000) or HRP-conjugated goat anti-mouse anti-IgG (Cell Signaling Technology, 7076s, 1:1000) antibodies. Next, the membrane was incubated with the ECL Western blotting detection reagents (Amersham Biosciences) for 1 minute, following the manufacturer's instructions. The protein bands were analyzed using ImageQuant LAS-4000 and dark box (FujiFilm). To determine the p10 levels and the IкB to GAPDH ratio, the immunoreactive bands were quantified using ImageJ software version 1.52a (NIH).

Quantitative PCR analysis. Total RNA was isolated from the wholelung tissue or cultured cells using TRIzol reagent (Invitrogen/Life Technologies). The purified RNA was treated with DNAse I, and $0.2 \mathrm{mg}$ RNA was reverse-transcribed into cDNA using TaqMan reverse transcription reagents (Applied Biosystems). The transcript levels of Nos2 (iNOS), Arg1, Retnla (Fizz1), Gas6, and Rsv- $g$ were determined by real-time PCR.
The fold difference in the expression levels between WT and Gas6-/mice was calculated using the $\Delta \Delta \mathrm{Ct}$ method, following the manufacturer's instructions (Applied Biosystems). GAPDH was used as an internal control. The fold changes in expression were calculated by comparing the target gene expression in the experimental group with that in the control mice and macrophages, which was assigned a value of 1 .

Statistics. Two-tailed Student's $t$ test (for 2-group analysis), 1-way ANOVA (for multiple-group analysis), and Gehan-Breslow-Wilcoxon test (comparisons of survival rates) were used to determine the statistical significances between the groups. All statistical analyses were performed using the GraphPad Prism software (version 7.0). A P value less than 0.05 was considered significant.

Study approval. All animal experiments were performed according to the guidelines of the Committees on Animal Handling and Ethical Regulations of the NIID, Japan. The research protocol was approved by the Institutional Animal Care and Use Committee of the Institute.

\section{Author contributions}

TS designed the study, performed the experiments, analyzed the data, and prepared the manuscript. AM and RO performed the experiments. Y. Takahashi contributed to the interpretation of the results with MA, SN, TI, KN, Y. Teraushi, TO, and MF, and prepared the manuscript.

\section{Acknowledgments}

We thank Sayaka Ogikubo for technical assistance. We also thank Russell Taichman (University of Michigan) and Carla Rothlin (Yale University) for providing $\mathrm{Gas}^{-/-}$and $\mathrm{Axl}^{-/-}$mice, respectively. This work was supported by the Takeda Science Foundation to TS and partly by the Japan Society for the Promotion of Science for Grant-in-Aid for Young Scientists (A) (16H06246) to TS and the Research Program on Emerging and Re-emerging Infectious Disease from the Japan Agency for Medical Research and Development (40106807) to TS, YT, and MA.

Address correspondence to: Takehiko Shibata, Tokyo Medical University, 6-1-1 Shinjuku, Shinjuku-ku, Tokyo, Japan, 160-8402. Phone: 81.3.3351.6141; Email: tshibata@tokyo-med.ac.jp.
1. Fernald GW, Almond JR, Henderson FW. Cellular and humoral immunity in recurrent respiratory syncytial virus infections. Pediatr Res. 1983;17(9):753-758.

2. Shi T, et al. Global, regional, and national disease burden estimates of acute lower respiratory infections due to respiratory syncytial virus in young children in 2015: a systematic review and modelling study. Lancet. 2017;390(10098):946-958.

3. Falsey AR, Hennessey PA, Formica MA, Cox C, Walsh EE. Respiratory syncytial virus infection in elderly and high-risk adults. $N$ Engl J Med. 2005;352(17):1749-1759.

4. García CG, et al. Risk factors in children hospitalized with RSV bronchiolitis versus non-RSV bronchiolitis. Pediatrics. 2010;126(6):e1453-e1460.

5. Nair H, et al. Global burden of acute lower respiratory infections due to respiratory syncytial virus in young children: a systematic review and meta-analysis. Lancet. 2010;375(9725):1545-1555.
6. Henderson FW, et al. A longitudinal study of respiratory viruses and bacteria in the etiology of acute otitis media with effusion. N Engl JMed. 1982;306(23):1377-1383.

7. Chonmaitree T, Owen MJ, Patel JA, Hedgpeth D, Horlick D, Howie VM. Effect of viral respiratory tract infection on outcome of acute otitis media. J Pediatr. 1992;120(6):856-862.

8. Suárez-Arrabal MC, et al. Nasopharyngeal bacterial burden and antibiotics: Influence on inflammatory markers and disease severity in infants with respiratory syncytial virus bronchiolitis. J Infect. 2015;71(4):458-469.

9. Weinberger DM, Klugman KP, Steiner CA, Simonsen L, Viboud C. Association between respiratory syncytial virus activity and pneumococcal disease in infants: a time series analysis of US hospitalization data. PLOS Med. 2015;12(1):e1001776.

10. Stark JM, Stark MA, Colasurdo GN, LeVine AM.
Decreased bacterial clearance from the lungs of mice following primary respiratory syncytial virus infection. JMed Virol. 2006;78(6):829-838.

11. Smith CM, et al. Respiratory syncytial virus increases the virulence of Streptococcus pneumoniae by binding to penicillin binding protein 1a. A new paradigm in respiratory infection. Am J Respir Crit Care Med. 2014;190(2):196-207.

12. Eckardt-Michel J, Lorek M, Baxmann D, Grunwald T, Keil GM, Zimmer G. The fusion protein of respiratory syncytial virus triggers p53-dependent apoptosis. J Virol. 2008;82(7):3236-3249.

13. Rowe HM, Meliopoulos VA, Iverson A, Bomme P, Schultz-Cherry S, Rosch JW. Direct interactions with influenza promote bacterial adherence during respiratory infections. Nat Microbiol. 2019;4(8):1328-1336.

14. Jochems SP, et al. Inflammation induced by influenza virus impairs human innate immune control of pneumococcus. Nat Immunol. 
2018;19(12):1299-1308.

15. Nakamura S, Davis KM, Weiser JN. Synergistic stimulation of type I interferons during influenza virus coinfection promotes Streptococcus pneumoniae colonization in mice. JClin Invest. 2011;121(9):3657-3665.

16. Shibata T, Habiel DM, Coelho AL, Kunkel SL, Lukacs NW, Hogaboam CM. Axl receptor blockade ameliorates pulmonary pathology resulting from primary viral infection and viral exacerbation of asthma. J Immunol. 2014;192(8):3569-3581.

17. Shibata T, Habiel DM, Coelho AL, Hogaboam $\mathrm{CM}$. Axl receptor blockade protects from invasive pulmonary aspergillosis in mice. J Immunol. 2014;193(7):3559-3565.

18. Fourgeaud L, et al. TAM receptors regulate multiple features of microglial physiology. Nature. 2016;532(7598):240-244.

19. van der Meer JH, van der Poll $T$, van ' $t$ Veer C. TAM receptors, Gas6, and protein S: roles in inflammation and hemostasis. Blood. 2014;123(16):2460-2469.

20. Rothlin CV, Ghosh S, Zuniga EI, Oldstone MB, Lemke G. TAM receptors are pleiotropic inhibitors of the innate immune response. Cell. 2007;131(6):1124-1136.

21. Sharif MN, et al. Twist mediates suppression of inflammation by type I IFNs and Axl. J Exp Med . 2006;203(8):1891-1901.

22. Duttweiler L, Nadal D, Frey B. Pulmonary and systemic bacterial co-infections in severe RSV bronchiolitis. Arch Dis Child. 2004;89(12):1155-1157.

23. Thorburn K, Harigopal S, Reddy V, Taylor N, van Saene HK. High incidence of pulmonary bacterial co-infection in children with severe respiratory syncytial virus (RSV) bronchiolitis. Thorax. 2006;61(7):611-615.

24. Hishiki $\mathrm{H}$, et al. Incidence of bacterial coinfection with respiratory syncytial virus bronchopulmonary infection in pediatric inpatients. J Infect Chemother. 2011;17(1):87-90.1.

25. Shibata T, et al. Role of growth arrest-specific gene 6 in the development of fungal allergic airway disease in mice. Am J Respir Cell Mol Biol. 2014;51(5):615-625.

26. Chen J, Carey K, Godowski PJ. Identification of Gas6 as a ligand for Mer, a neural cell adhe- sion molecule related receptor tyrosine kinase implicated in cellular transformation. Oncogene. 1997;14(17):2033-2039.

27. Fisher PW, et al. A novel site contributing to growth-arrest-specific gene 6 binding to its receptors as revealed by a human monoclonal antibody. Biochem J. 2005;387(Pt 3):727-735.

28. Nagata K, et al. Identification of the product of growth arrest-specific gene 6 as a common ligand for Axl, Sky, and Mer receptor tyrosine kinases. J Biol Chem. 1996;271(47):30022-30027.

29. Misharin AV, et al. Monocyte-derived alveolar macrophages drive lung fibrosis and persist in the lung over the life span. JExp Med. 2017;214(8):2387-2404.

30. Holland SJ, et al. R428, a selective small molecule inhibitor of Axl kinase, blocks tumor spread and prolongs survival in models of metastatic breast cancer. Cancer Res. 2010;70(4):1544-1554.

31. Xu F, et al. Modulation of the inflammatory response to Streptococcus pneumoniae in a model of acute lung tissue infection. Am J Respir Cell Mol Biol. 2008;39(5):522-529.

32. Shirey KA, et al. Control of RSV-induced lung injury by alternatively activated macrophages is IL-4R $\alpha$-, TLR4-, and IFN- $\beta$-dependent. Mucosal Immunol. 2010;3(3):291-300.

33. Wang $\mathrm{N}$, Liang $\mathrm{H}$, Zen $\mathrm{K}$. Molecular mechanisms that influence the macrophage m1-m2 polarization balance. Front Immunol. 2014;5:614.

34. Cooper AM, Dalton DK, Stewart TA, Griffin JP, Russell DG, Orme IM. Disseminated tuberculosis in interferon- $\gamma$ gene-disrupted mice. J Exp Med.1993;178(6):2243-2247.

35. Buchmeier NA, Schreiber RD. Requirement of endogenous interferon-gamma production for resolution of Listeria monocytogenes infection. Proc Natl Acad Sci U S A. 1985;82(21):7404-7408.

36. Yamada $M$, et al. Interferon- $\gamma$ production by neutrophils during bacterial pneumonia in mice. $\mathrm{Am}$ J Respir Crit Care Med. 2011;183(10):1391-1401.

37. Gomez JC, et al. Mechanisms of interferon- $\gamma$ production by neutrophils and its function during Streptococcus pneumoniae pneumonia. Am J Respir Cell Mol Biol. 2015;52(3):349-364.

38. Nepal S, et al. STAT6 induces expression of Gas6 in macrophages to clear apoptotic neutrophils and resolve inflammation. Proc Natl Acad Sci
U S A. 2019;116(33):16513-16518.

39. Han J, et al. Autophagy induced by AXL receptor tyrosine kinase alleviates acute liver injury via inhibition of NLRP3 inflammasome activation in mice. Autophagy. 2016;12(12):2326-2343.

40. Ptaschinski C, et al. RSV-induced H3K4 demethylase KDM5B leads to regulation of dendritic cell-derived innate cytokines and exacerbates pathogenesis in vivo. PLoS Pathog. 2015;11(6):e1004978.

41. Saravia J, et al. Respiratory syncytial virus disease is mediated by age-variable IL-33. PLoS Pathog. 2015;11(10):e1005217.

42. Stier MT, et al. Respiratory syncytial virus infection activates IL-13-producing group 2 innate lymphoid cells through thymic stromal lymphopoietin. J Allergy Clin Immunol. 2016;138(3):814-824.e11.

43. Shibata T, Hogaboam CM. Gas6'ing the innate immune response during experimental asthma. Ann Am Thorac Soc. 2014;11(Suppl 5):S303-S305.

44. Yokoyama $\mathrm{T}$, et al. A necrotic lung ball caused by co-infection with Candida and Streptococcus pneumoniae. Infect Drug Resist. 2011;4:221-224.

45. Barthelemy A, et al. Influenza A virus-induced release of interleukin-10 inhibits the anti-microbial activities of invariant natural killer $\mathrm{T}$ cells during invasive pneumococcal superinfection Mucosal Immunol. 2017;10(2):460-469.

46. Didierlaurent A, et al. Sustained desensitization to bacterial Toll-like receptor ligands after resolution of respiratory influenza infection. J Exp Med.2008;205(2):323-329.

47. Shahangian A, et al. Type I IFNs mediate development of postinfluenza bacterial pneumonia in mice. JClin Invest. 2009;119(7):1910-1920.

48. Sun K, Metzger DW. Inhibition of pulmonary antibacterial defense by interferon-gamma during recovery from influenza infection. Nat Med. 2008;14(5):558-564.

49. Tripp RA, Jones LP, Haynes LM, Zheng H, Murphy PM, Anderson LJ. CX3C chemokine mimicry by respiratory syncytial virus $\mathrm{G}$ glycoprotein. Nat Immunol. 2001;2(8):732-738.

50. Shibata T, Ato M. A critical role of Gas6/Axl signal in allergic airway responses during RSV vaccine-enhanced disease. Immunol Cell Biol. 2017;95(10):906-915. 